\title{
Dispersion for Schrödinger Operators with One-gap Periodic Potentials on $\mathbb{R}^{1}$
}

\author{
Kaihua Cai
}

Communicated by Terence Tao, received September 12, 2005.

\begin{abstract}
We prove $t^{-\frac{1}{4}}$-decay for the solutions of the 1-dim Schrodinger equation with a one-gap periodic potential as $t \rightarrow+\infty$. Generically, one has $t^{-\frac{1}{3}}$-decay and this decay is optimal. Our approach is to analyze the stationary phase in the Schrödinger evolution as an integral operator.
\end{abstract}

\section{Contents}

1. Introduction $\quad 71$

2. Preliminaries $\quad 75$

3. Analysis of $K_{1}\left(t, x, x^{\prime}\right) \quad 78$

4. Analysis of $K_{2}\left(t, x, x^{\prime}\right) \quad 81$

5. Optimality of the decay factor $\quad 87$

6. Appendix 90

$\begin{array}{ll}\text { References } & 91\end{array}$

\section{Introduction}

The dispersive property of the Schrödinger equation with time-independent potentials

$$
\frac{1}{i} \partial_{t} \psi=-\Delta \psi+V(x) \psi
$$

has played an important role in the recent development of the NLS equations. It usually takes the following form:

1991 Mathematics Subject Classification. Primary 35J10; Secondary 35.

Key words and phrases. Schrödinger equation, dispersive property, stationary phase, one-gap periodic potential.

The author feels deeply grateful to his advisor Wilhelm Schlag for his guidance. Also the author thanks Irina Nenciu for reading a preliminary version of this paper.

(C)2006 International Press 


$$
\left\|e^{i H t} P_{a . c .}(H) \varphi\right\|_{\infty}<C t^{-\frac{n}{2}}\|\varphi\|_{L^{1}\left(\mathbb{R}^{n}\right)}
$$

where $H=-\Delta+V(x), n$ is the space dimension and $P_{a . c .}(H)$ is the projection onto the absolutely continuous spectral subspace. $P_{\text {a.c. }}(H)$ is necessary here since the bound states do not disperse over time. To obtain (1.2), two kinds of conditions on $H$ are usually assumed. The first condition is spectrum assumption at zero energy: $H$ has neither an eigenvalue nor a resonance at zero energy. If $H$ has an eigenvalue or a resonace at zero energy, analogy of (1.2) can be obtained with the decay factor $t^{-\frac{n}{2}-1}$. The second condition is an integrability condition on $V$; i.e., $V$ lies in some $L^{p}\left(\mathbb{R}^{n}\right)$ space or $|V(x)| \leq C(1+|x|)^{-\sigma}$ for some positive $\sigma$. For the detail results of this aspect, among all we just list the following papers: $[\mathbf{R}],[\mathbf{J K}]$, $[\mathbf{J S S}],[\mathbf{Y a}],[\mathbf{R S}],[\mathbf{G S}],[\mathbf{W e 2}]$ and $[\mathbf{G o}]$.

In this article, we explore the dispersive property of $H$ under the assumption that $V(x)$ is real-valued, periodic and defined on the real line. In this case, the differential equation

$$
-y^{\prime \prime}(x)+V(x) y(x)=E y(x)
$$

defined on the real line is known as Hill's equation.

One of our motivations to study this case comes from the research of the random Schrödinger operators. Let

$$
H_{\omega}=-\frac{d^{2}}{d x^{2}}+\sum_{n \in \mathbb{Z}} q_{n}(\omega) f(x-n),
$$

where the single-site potential $f \in L^{1}$ is real-valued, supported in $[-1 / 2,1 / 2]$. The coupling constants $q_{n}$ are i.i.d. random variables on a complete probability space. We assume that the support of their common distribution contains at least two points. Note otherwise the potential is deterministic and periodic. Under these assumptions, almost surely $H_{\omega}$ has pure point spectrum and all eigenfunctions decay exponentially at $\pm \infty$. The philosophy behind is that when the waves pass through each period, there are reflection and transmission taking place. The randomness of the potential over each period guarantees that reflection is strong enough for each wave packet to localize in space. This scenario is quite different from the case of the period potential. With fixed reflection and transmission coefficients, no eigenfunction can be localized in space. It is well-known that the spectrum of $H=-\left(d^{2} / d x^{2}\right)+V(x)$ is purely absolutely continuous, where $V(x)$ is periodic. Therefore, it is natural to ask what kind of dispersive property $H=-\left(d^{2} / d x^{2}\right)+V$ may have. In [DSS], Anderson localization was also established almost surely for the operator $H_{\omega}=-\frac{d^{2}}{d x^{2}}+V_{\text {per }}+V_{\omega}$, where $V_{\text {per }}$ has period 1 and $V_{\omega}=\sum_{n \in \mathbb{Z}} q_{n}(\omega) f(x-n)$. For details and related results, see the reference of $[\mathbf{D S S}]$.

Suppose $V(x)=V(x+2 \omega)$. The spectrum of the Schrödinger operator $H=$ $-\left(d^{2} / d x^{2}\right)+V(x)$ acting on $L^{2}\left(\mathbb{R}^{1}\right)$ is a union of intervals carrying purely absolutely continuous spectrum. The absence of point and singular spectrum suggests the dispersion of the solution.

Dispersive properties of the wave equation with periodic potentials have been studied. Suppose $U(t) \psi_{0}=\psi(t, x)$ is the solution of $\partial_{t}^{2} \psi=-H \psi$, satisfying 
$\psi(0, x)=0$ and $\partial_{t} \psi(0, x)=\psi_{0}$. Denote $P_{n}(H)$ is the spectral projection onto the $n$-th interval in the spectrum of $H$. In $[\mathbf{K o}]$, the asymptotic behavior of the evolution operator $U(t) P_{n}(H)\left(x, x^{\prime}\right)$ is obtained as $t \rightarrow 0$ when $\left(x-x^{\prime}, t\right)$ is away from the $n$-th light cone. The disadvantage of working with general periodic potentials is losing control of the degeneracy in critical region. Also it is delicate to sum up over all the spectral intervals to get an estimate for the evolution $U(t)$.

To avoid these difficulties with the general potentials, we will illustrate the dispersive phenomenon of (1.1) for a special analytic potential, whose spectrum is a union of two intervals (bands); namely, all gaps but one are degenerate. It is known that one-gap potentials must be elliptic functions $([\mathbf{H o}])$. With such a potential, we rewrite Eq.(1.3) as

$$
y^{\prime \prime}(x)-2 \wp\left(x+\omega_{3}\right) y(x)=-E y(x),
$$

where $\wp(z)$ is the Weierstrass elliptic function with periods $2 \omega_{1}, 2 \omega_{3}$, satisfying the following differential equations:

$$
\begin{array}{r}
\wp^{\prime}(a)^{2}=4 \wp^{3}(a)-g_{2} \wp(a)-g_{3}, \\
\wp^{\prime \prime}(a)=6 \wp^{2}(a)-\frac{g_{2}}{2} .
\end{array}
$$

where $g_{2}, g_{3}$ are the invariants of $\wp(z)$ defined by (6.1). Eq.(1.4), known as Lamé's equation, arises from the theory of the potential of an ellipsoid $([\mathbf{W W}],[\mathbf{E r}])$. We assume $\omega_{1}=\omega>0, \omega_{3}=i \omega^{\prime}$ and $\omega^{\prime}>0$ to guarantee that $\wp\left(x+\omega_{3}\right)$ is real-valued for $x \in \mathbb{R}$. If we choose the potential in $(1.4)$ to be $n(n+1) \wp$, instead of $2 \wp(n$ any positive integer), then the spectrum of Lamé's equation consists of $n+1$ bands $([\mathbf{M W}])$.

Eigenfunctions of (1.4) are expressed in terms of the Weierstrass $\sigma$-function and $\zeta$-function $([\mathbf{K M}],[\mathbf{G S S}])$ as follows:

$$
f_{a}(x)=\frac{\sigma\left(x+i \omega^{\prime}+a\right)}{\sigma\left(x+i \omega^{\prime}\right)} e^{-\zeta(a) x-\zeta\left(i \omega^{\prime}\right) a}
$$

where the energy

$$
E=-\wp(a)
$$

Some basic properties of Weierstrass functions are listed in the appendix. (1.7) can be verified by noticing that $([\mathbf{W W}])$

$$
f_{a}^{\prime}(x)=\left(\zeta\left(x+\omega_{3}+a\right)-\zeta\left(x+\omega_{3}\right)-\zeta(a)\right) f_{a}(x)
$$

and

$$
(\zeta(x+y)-\zeta(x)-\zeta(y))^{2}=\wp(x+y)+\wp(x)+\wp(y) .
$$

$f_{a}$ is periodic when $a$ is one of the half periods $\omega_{1}, \omega_{2}=\omega_{1}+\omega_{3}$ or $\omega_{3}$. $f_{-a}$ and $f_{a}$ are the two Floquet-type solutions of (1.4). We write

where

$$
m_{a}(x)=\frac{\sigma\left(x+i \omega^{\prime}+a\right)}{\sigma\left(x+i \omega^{\prime}\right)} e^{-a \zeta\left(i \omega^{\prime}\right)-a \frac{x}{\omega} \zeta(\omega)}
$$

is periodic with period $2 \omega$. Denote 


$$
\Sigma=\left[-\wp\left(\omega_{1}\right),-\wp\left(\omega_{2}\right)\right] \cup\left[-\wp\left(\omega_{3}\right),+\infty\right),
$$

and the quasimomentum

$$
k(a)=i \omega^{-1}(\omega \zeta(a)-a \zeta(\omega))
$$

is real-valued for $E \in \Sigma$. $f_{a}$ is bounded when $E \in \Sigma$ and is unbounded otherwise, which implies that $\Sigma$ is the spectrum of (1.4) ([MW]).

Our goal is to give a dispersive estimate similar to (1.2) for the following

$$
\begin{aligned}
\frac{1}{i} \partial_{t} \psi(x, t) & =-\frac{d^{2}}{d x^{2}} \psi(x, t)+2 \wp\left(x+\omega_{3}\right) \psi(x, t), \\
\psi(x, 0) & =\psi_{0}(x) .
\end{aligned}
$$

We assume that $\psi_{0} \in L^{1}(\mathbb{R})$ and denote the solution at time $t$ as $U(t) \psi_{0}$.

THEOREM 1.1. Generically, for almost all $\omega, \omega^{\prime} \in \mathbb{R}$, there exists a constant $C>0$ such that for $t>1$

$$
\left\|U(t) \psi_{0}\right\|_{L^{\infty}(\mathbb{R})}<C t^{-\frac{1}{3}}\left\|\psi_{0}\right\|_{L^{1}(\mathbb{R})} .
$$

Moreover, for all nonzero $\omega, \omega^{\prime} \in \mathbb{R}$, there exists a constant $C>0$ such that for $t>1$

$$
\left\|U(t) \psi_{0}\right\|_{L^{\infty}(\mathbb{R})}<C t^{-\frac{1}{4}}\left\|\psi_{0}\right\|_{L^{1}(\mathbb{R})} .
$$

(1.11) is optimal in the sense that for any nonzero $\omega, \omega^{\prime} \in \mathbb{R}$, there exist constants $c>0$ and $T>0$, depending only on $\omega, \omega^{\prime}$ such that for $t>T$

$$
\sup _{\psi_{0}:\left\|\psi_{0}\right\|_{L^{1}(\mathbb{R})}=1}\left\|U(t) \psi_{0}\right\|_{L^{\infty}(\mathbb{R})}>c t^{-\frac{1}{3}}
$$

Corollary 1.2. Suppose that $(\zeta(\omega) / \omega)^{2} \leq g_{2} / 12$. Then for $t>1$,

$$
\left\|U(t) \psi_{0}\right\|_{L^{\infty}}<C t^{-\frac{1}{3}}\left\|\psi_{0}\right\|_{L^{1}(R)} .
$$

We require $t>1$ only to exclude $t \rightarrow 0$. The decay rates $t^{-\frac{1}{3}}$ and $t^{-\frac{1}{4}}$ are different from $t^{-\frac{1}{2}}$ in (1.2) because phase function is non-quadratic, which is a natural outcome of the periodic potential. The decay factor $t^{-\frac{1}{3}}$ as $t \rightarrow \infty$ has appeared in the analysis of the Modified KdV equation ([DZ]), where the nonlinear phase of the main term is cubic. In our case, the analytic phase function, roughly speaking, satisfies a cubic relation up to a change of variables. This cubic relation comes from the differential equations satisfied by the Weierstrass $\wp$ function . We denote $P(x)$ to be the real-coefficient cubic polynomial

$$
2 x^{3}+\frac{6 \zeta(\omega)}{\omega} x^{2}+\frac{g_{2}}{2} x+g_{3}-\frac{g_{2} \zeta(\omega)}{2 \omega} .
$$

We shall prove (1.11) under the assumption that

$$
P(x) \text { has no double root in }\left(-\infty, \wp\left(\omega_{3}\right)\right] \text {. }
$$


If (1.15) does not hold, then we shall prove (1.12). In this case, by Lemma 2.4, $P(x)$ has no root of degree 3 . Our proof implies that (1.12) is optimal in the sense stated in Theorem 1.1. However, we are unable to give an explicit example such that $P(x)$ does have a double root in $\left(-\infty, \wp\left(\omega_{3}\right)\right]$.

Finally, we prove that assumption (1.15) holds for almost all $\omega, \omega^{\prime} \in \mathbb{R}$.

\section{Preliminaries}

The evolution operator $U(t)=e^{i t H}$ can be written out via the generalised Fourier transform ([We], $[\mathbf{W e 2}]$ ) or via the spectral measure $([\mathbf{G S}])$. Since the spectrum is purely absolutely continuous, we will use the latter one and write the kernel of $U(t)$ as

$$
K\left(t, x, x^{\prime}\right)=\int_{\Sigma} e^{i t E} P_{a . c .}\left(E, x, x^{\prime}\right) d E
$$

Namely,

$$
\psi(x, t)=\int_{\Sigma} \int_{\mathbb{R}} e^{i t E} P_{a . c .}\left(E, x, x^{\prime}\right) \psi_{0}\left(x^{\prime}\right) d x^{\prime} d E .
$$

The absolutely continuous spectral projection is

$$
P_{a . c .}\left(E, x, x^{\prime}\right)=\frac{1}{2 \pi i}\left[(H-(E+i 0))^{-1}\left(x, x^{\prime}\right)-(H-(E-i 0))^{-1}\left(x, x^{\prime}\right)\right],
$$

and by definition

$$
(H-(E \pm i 0))^{-1}=\lim _{\epsilon \rightarrow 0^{+}}(H-(E \pm i \epsilon))^{-1},
$$

which can be expressed by $f_{a}$ and $f_{-a}$. Hence, we obtain for $x>x^{\prime}$

$$
\begin{aligned}
K\left(t, x, x^{\prime}\right) & =\int_{\Sigma} e^{i t E}\left(f_{-a}\left(x^{\prime}\right) f_{a}(x)+f_{-a}(x) f_{a}\left(x^{\prime}\right)\right) \frac{d E}{W(E)} \\
(2.1) & =\int_{\Sigma} e^{i t E}\left(e^{i k(a)\left(x-x^{\prime}\right)} m_{-a}\left(x^{\prime}\right) m_{a}(x)+e^{-i k(a)\left(x-x^{\prime}\right)} m_{-a}(x) m_{a}\left(x^{\prime}\right)\right) \frac{d E}{W(E)},
\end{aligned}
$$

where $W(E)=W(a)=W\left(f_{a}, f_{-a}\right)=f_{a} f_{-a}^{\prime}-f_{a}^{\prime} f_{-a}$, called the Wronskian of $f_{a}, f_{-a}$, is independent of $x$.

Because the spectral projection $P_{a . c .}$ is self-adjoint, $P_{a . c .}\left(E, x, x^{\prime}\right)=\overline{P_{a . c .}\left(E, x^{\prime}, x\right)}$. Therefore, when $x<x^{\prime}$

$$
K\left(t, x, x^{\prime}\right)=\int_{\Sigma} e^{i t E}\left(\overline{f_{-a}}\left(x^{\prime}\right) \overline{f_{a}}(x)+\overline{f_{-a}}(x) \overline{f_{a}}\left(x^{\prime}\right)\right) \frac{d E}{\bar{W}(E)} .
$$

The proof of (1.11) and (1.12) shall be reduced to proving

$$
\sup _{x, x^{\prime}}\left|K\left(t, x, x^{\prime}\right)\right|<C t^{-\frac{1}{3}} \text { and } C t^{-\frac{1}{4}} \text {. }
$$

LEMMA 2.1.

$$
\frac{d E}{W(E)}=-\frac{\sigma^{2}\left(i \omega^{\prime}\right)\left(\wp\left(i \omega^{\prime}\right)-\wp(a)\right)}{\sigma\left(i \omega^{\prime}+a\right) \sigma\left(i \omega^{\prime}-a\right)} d a
$$


Proof. It follows from Eq. (1.8) that

$$
W\left(f_{a}, f_{-a}\right)=f_{a}(x) f_{-a}(x)\left(\zeta\left(x+\omega_{3}-a\right)+2 \zeta(a)-\zeta\left(x+\omega_{3}+a\right)\right) .
$$

Since $W\left(f_{a}, f_{-a}\right)$ is independent of $x$, we set $x=0$ and obtain

$$
W\left(f_{a}, f_{-a}\right)=f_{a}(0) f_{-a}(0)\left(\zeta\left(\omega_{3}-a\right)+2 \zeta(a)-\zeta\left(\omega_{3}+a\right)\right) .
$$

By the addition formula for Weierstrass functions $([\mathbf{A k}], \S 15)$

we have

$$
\zeta(u+v)-\zeta(u-v)-2 \zeta(v)=-\frac{\wp^{\prime}(v)}{\wp(u)-\wp(v)},
$$

$$
W(E)=\frac{\sigma\left(i \omega^{\prime}+a\right) \sigma\left(i \omega^{\prime}-a\right)}{\sigma^{2}\left(i \omega^{\prime}\right)} \frac{\wp^{\prime}(a)}{\wp\left(i \omega^{\prime}\right)-\wp(a)} .
$$

Therefore, (2.3) follows from $E=-\wp(a)$.

REMARK 2.2. Because $\wp^{\prime}\left(i \omega^{\prime}\right)=0$ and zeroes of $\sigma$ are the lattice points $\left\{n_{1} 2 \omega_{1}+n_{2} 2 \omega_{3}: n_{1}, n_{2} \in Z\right\}$, all of which are of degree 1 , it follows that $\frac{\wp\left(i \omega^{\prime}\right)-\wp(a)}{\sigma\left(i \omega^{\prime}+a\right) \sigma\left(i \omega^{\prime}-a\right)}$ is bounded and smooth when $a \rightarrow i \omega^{\prime}$.

Also, it is clear that $\frac{\wp\left(i \omega^{\prime}\right)-\wp(a)}{\sigma\left(i \omega^{\prime}+a\right) \sigma\left(i \omega^{\prime}-a\right)}=O\left(a^{-2}\right)$ when $a \rightarrow 0$, and that $\frac{\wp\left(i \omega^{\prime}\right)-\wp(a)}{\sigma\left(i \omega^{\prime}+a\right) \sigma\left(i \omega^{\prime}-a\right)}$ and its $\partial_{a}$-derivatives are bounded on $\left[\omega_{1}, \omega_{2}\right]$, where $\left[\omega_{1}, \omega_{2}\right]$ denotes the set

$$
\left\{\lambda \omega_{1}+(1-\lambda) \omega_{2}: \lambda \in[0,1]\right\}
$$

By (1.9), $m_{a}, m_{-a}$ and their $\partial_{a}$-derivatives are bounded uniformly for $a \in\left[\omega_{1}, \omega_{2}\right] \cup$ $\left[0, \omega_{3}\right], x \in \mathbb{R}$.

Since $\Sigma$ is a union of two intervals, we shall decompose the integral of $K\left(t, x, x^{\prime}\right)$ into two parts. Namely,

where

$$
K\left(t, x, x^{\prime}\right)=K_{1}\left(t, x, x^{\prime}\right)+K_{2}\left(t, x, x^{\prime}\right),
$$

$$
\begin{gathered}
K_{1}\left(t, x, x^{\prime}\right)=\int_{-\wp\left(\omega_{1}\right)}^{-\wp\left(\omega_{2}\right)} e^{i t E} P_{a . c .}\left(E, x, x^{\prime}\right) d E, \\
K_{2}\left(t, x, x^{\prime}\right)=\int_{-\wp\left(\omega_{3}\right)}^{+\infty} e^{i t E} P_{a . c .}\left(E, x, x^{\prime}\right) d E .
\end{gathered}
$$

Before we proceed to analyze $K_{1}\left(t, x, x^{\prime}\right)$ and $K_{2}\left(t, x, x^{\prime}\right)$, we prove two technical lemmas.

LEMMA 2.3. Let $F(x)$ be a real-valued and smooth function on $(a, b)$,

(1) Suppose $\left|F^{\prime}(x)\right| \geq \epsilon,\left|F^{\prime \prime}(x)\right| \leq M$ for all $x \in(a, b)$, then

$$
\left|\int_{a}^{b} e^{-i t F(x)} \psi(x) d x\right| \leq c|t|^{-1}\left[|\psi(b)|+\int_{a}^{b}\left(\left|\psi^{\prime}(x)\right|+|\psi(x)|\right) d x\right],
$$

where $c$ depends on $M$ and $\epsilon$.

(2) Suppose $k \geq 2, k \in \mathbb{Z}$ and $\left|F^{(k)}(x)\right| \geq \epsilon$ for all $x \in(a, b)$, then

$$
\left|\int_{a}^{b} e^{-i t F(x)} \psi(x) d x\right| \leq c \epsilon^{-\frac{1}{k}}|t|^{-\frac{1}{k}}\left[|\psi(b)|+\int_{a}^{b}\left|\psi^{\prime}(x)\right| d x\right],
$$

where $c$ depends on $k$. 
The first part of Lemma 2.3 follows from integration by parts. The second part is proved in $[\mathbf{S t}]$ (p. 334).

Lemma 2.4. Let $e_{j}=\wp\left(\omega_{j}\right), j=1,2,3$. Then $P(x)$ has a unique simple root in $\left[e_{2}, e_{1}\right]$, and $P\left(e_{j}\right), j=1,2,3$, are nonzero. Also $P(x)$ has no root of degree 3 in $\mathbb{R}$. Moreover, $-\frac{\zeta(\omega)}{\omega} \in\left(e_{3}, e_{2}\right)$.

Proof. $P(x)=0$ if and only if $4 x^{3}-g_{2} x-g_{3}=\left(6 x^{2}-\frac{g_{2}}{2}\right)\left(x+\frac{\zeta(\omega)}{\omega}\right)$. Denote $p_{1}(x)=4 x^{3}-g_{2} x-g_{3}$ and $p_{2}(x)=\left(6 x^{2}-\frac{g_{2}}{2}\right)\left(x+\frac{\zeta(\omega)}{\omega}\right)$. We shall examine the roots of $p_{1}(x)$ and $p_{2}(x)$ on the real line.

It follows from Eq. (6.2) that $p_{1}(x)=4\left(x-e_{1}\right)\left(x-e_{2}\right)\left(x-e_{3}\right)$, where $e_{j}=\wp\left(\omega_{j}\right)$, $j=1,2,3$. Because there is no quadratic term in $p_{1}(x), e_{1}+e_{2}+e_{3}=0$. Since $e_{3}<e_{2}<e_{1}$, we have $e_{3}<0<e_{1}$.

Observe that $\wp^{\prime \prime}\left(\omega_{1}\right)>0, \wp^{\prime \prime}\left(\omega_{2}\right)<0$ and $\wp^{\prime \prime}\left(\omega_{3}\right)>0$, and by Eq (1.6), we obtain

$$
\wp\left(\omega_{2}\right)^{2}<\frac{g_{2}}{12}<\min \left\{\wp\left(\omega_{1}\right)^{2}, \wp\left(\omega_{3}\right)^{2}\right\} .
$$

Now we shall prove $\frac{\zeta(\omega)}{\omega} \in\left(-e_{2},-e_{3}\right)$. Indeed, let $y_{1}(x, E)$ and $y_{2}(x, E)$ be the solutions of (1.4) which satisfy

$$
y_{1}(0, E)=y_{2}^{\prime}(0, E)=1, \quad y_{1}^{\prime}(0, E)=y_{2}(0, E)=0 .
$$

And we introduce the discriminant $\Delta(E)=y_{1}(2 \omega, E)+y_{2}^{\prime}(2 \omega, E)$.

Recall $a$ and $E$ are related by $E=-\wp(a)$, and as $E \rightarrow+\infty$ on the real line, $a \rightarrow 0$ on the positive imaginary axis. Therefore $i \zeta(a)$ and $k(a)$ go to $+\infty$ on the real line when $E \rightarrow+\infty$.

By Lemma 2.1 of $[\mathbf{G T}], \Delta(E)=2 \cos k(a)$ and $k(E)=k(a(E))$ is the conformal map from the upper half plane to a slit quarter plane $\Omega=\{\Re z>0, \Im z>0\} \backslash T$, with the slit $T=\left\{\frac{\pi}{2 \omega}+i y: 0<y \leq h\right\}$, where $h$ is some positive real number. Moreover, $k\left(-e_{1}\right)=0$ and $k\left(-e_{2}\right)=k\left(-e_{3}\right)=\frac{\pi}{2 \omega}$.

Denote $Q_{0}$ to be the pre-image of the tip $\frac{\pi}{2 \omega}+i h$ of the slit $T$ under the map $k(E)$. Then $-\wp\left(\omega_{2}\right)<Q_{0}<-\wp\left(\omega_{3}\right)$, and $k(E)$ sends $\left[-\wp\left(\omega_{2}\right), Q_{0}\right]$ to $\left[\frac{\pi}{2 \omega}, \frac{\pi}{2 \omega}+i h\right]$, and $\left[Q_{0},-\wp\left(\omega_{3}\right)\right]$ to $\left[\frac{\pi}{2 \omega}+i h, \frac{\pi}{2 \omega}\right]$ respectively. Thus when $E \in\left(-\wp\left(\omega_{2}\right), Q_{0}\right)$, $\frac{1}{i} \partial_{E} k(E) \geq 0$. We observe that

$$
\partial_{E} k(E)=\frac{1}{-\wp^{\prime}(a)} \partial_{a} k(a)=\frac{i}{\wp^{\prime}(a)}\left(\frac{\zeta(\omega)}{\omega}+\wp(a)\right),
$$

which implies that

$$
\frac{\wp(a)+\zeta(\omega) / \omega}{\wp^{\prime}(a)} \geq 0
$$

Since $\wp^{\prime}(a)>0$ when $a \in\left(\omega_{3}, \omega_{2}\right)$, we conclude that $E=-\wp(a) \leq \zeta(\omega) / \omega$ for any $E \in\left(-\wp\left(\omega_{2}\right), Q_{0}\right)$. Hence, $Q_{0} \leq \zeta(\omega) / \omega$. On the other hand, $\frac{1}{i} \partial_{E} k(E) \leq 0$ when $E \in\left(Q_{0},-\wp\left(\omega_{3}\right)\right)$. Following the similar argument, $Q_{0} \geq \zeta(\omega) / \omega$. Therefore $\zeta(\omega) / \omega=Q_{0} \in\left(-\wp\left(\omega_{2}\right),-\wp\left(\omega_{3}\right)\right)$.

In fact, $k(E)$ maps $E=\frac{\zeta(\omega)}{\omega}$ to the tip $\frac{\pi}{2 \omega}+i h$ of the slit $T$ and $\Delta(E)$ reaches its minimum at $E=\frac{\zeta(\omega)}{\omega}$. 
The three roots of $p_{2}(x)$ are $\pm \sqrt{\frac{g 2}{12}}$ and $-\frac{\zeta(\omega)}{\omega}$. From the above analysis, we have that $\sqrt{\frac{g_{2}}{12}} \in\left(e_{2}, e_{1}\right)$ and $-\sqrt{\frac{g_{2}}{12}},-\frac{\zeta(\omega)}{\omega} \in\left(e_{3}, e_{2}\right)$, which implies $p_{2}\left(e_{1}\right)>0$ and $p_{2}\left(e_{2}\right)<0$. Hence $P(x)$ has either one or three zeroes in $\left(e_{2}, e_{1}\right)$ and clearly $P\left(e_{j}\right), j=1,2,3$, are nonzero.

To verify that $P(x)$ has no root of degree 3 , we consider

$$
P^{\prime}(x)=6 x^{2}+12 \frac{\zeta(\omega)}{\omega} x+\frac{g_{2}}{2} .
$$

The minimum of $P^{\prime}(x)$ is reached at $x=-\frac{\zeta(\omega)}{\omega} \in\left(e_{3}, e_{2}\right)$ and is equal to $\frac{g_{2}}{2}-$ $6\left(\frac{\zeta(\omega)}{\omega}\right)^{2}$. Notice that $-\frac{\zeta(\omega)}{\omega}<e_{2}<\sqrt{\frac{g_{2}}{12}}$ always holds.

If $-\frac{\zeta(\omega)}{\omega}>-\sqrt{\frac{g 2}{12}}$, then $P^{\prime}(x)>0$ holds for all $x \in \mathbb{R}$. $P(x)$ has no root of degree greater or equal to 2 .

If $-\frac{\zeta(\omega)}{\omega}=-\sqrt{\frac{g_{2}}{12}}$, then $P^{\prime}(x)$ has a double root $-\frac{\zeta(\omega)}{\omega} \in\left(e_{3}, e_{2}\right)$. Since $P(x)$ has a root in $\left(e_{2}, e_{1}\right)$, we conclude that $P(x)$ has no root of degree 3 .

If $-\frac{\zeta(\omega)}{\omega}<-\sqrt{\frac{g_{2}}{12}}$, then $P^{\prime}(x)$ has no double root. Hence $P(x)$ has no root of degree 3 on the whole real line.

If $P(x)$ has three zeroes in $\left(e_{2}, e_{1}\right)$, then $P^{\prime}(x)$ has two roots in $\left(e_{2}, e_{1}\right)$, which is impossible because $-\frac{\zeta(\omega)}{\omega}<e_{2}$. Therefore, $P(x)$ has unique simple root in $\left(e_{2}, e_{1}\right)$.

\section{Analysis of $K_{1}\left(t, x, x^{\prime}\right)$}

We first consider $K_{1}\left(t, x, x^{\prime}\right)$. We proceed by making the following observation:

Lemma 3.1. Let $b=2 \omega_{2}-a$ for $a \in\left[\omega_{1}, \omega_{2}\right]$. Write $W(a)=W\left(f_{a}, f_{-a}\right)$. Then for $x, x^{\prime} \in \mathbb{R}$

$$
\frac{f_{a}\left(x^{\prime}\right) f_{-a}(x)}{W(a)}=-\frac{f_{b}(x) f_{-b}\left(x^{\prime}\right)}{W(b)} .
$$

Proof. It is clear that $\wp(a)=\wp(b)$ and $\wp^{\prime}(a)=-\wp^{\prime}(b)$. We prove (3.1) by direct calculation. By definition,

$$
f_{a}\left(x^{\prime}\right) f_{-a}(x)=\frac{\sigma\left(x^{\prime}+\omega_{3}+a\right) \sigma\left(x+\omega_{3}-a\right)}{\sigma\left(x+\omega_{3}\right) \sigma\left(x^{\prime}+\omega_{3}\right)} e^{\zeta(a)\left(x-x^{\prime}\right)} .
$$

By Eq.(6.4) and (6.5), this equals

$$
\frac{\sigma\left(x^{\prime}+\omega_{3}-b\right) \sigma\left(x+\omega_{3}+b\right)}{\sigma\left(x+\omega_{3}\right) \sigma\left(x^{\prime}+\omega_{3}\right)} e^{\zeta(b)\left(x^{\prime}-x\right)} e^{4 \eta_{3}\left(\omega_{3}-b\right)}=f_{b}(x) f_{-b}\left(x^{\prime}\right) e^{4 \eta_{3}\left(\omega_{3}-b\right)} .
$$

Also by Eq.(2.4) and (6.5),

$$
W(a)=\frac{\sigma\left(i \omega^{\prime}-b\right) \sigma\left(i \omega^{\prime}+b\right) \exp \left(4 \eta_{3}\left(\omega_{3}-b\right)\right)}{\sigma^{2}\left(i \omega^{\prime}\right)} \frac{-\wp^{\prime}(b)}{\wp\left(\omega_{3}\right)-\wp(b)}=-W(b) e^{4 \eta_{3}\left(\omega_{3}-b\right)} .
$$

Combining them, (3.1) follows.

It follows from Lemma 3.1 that

$$
\int_{\omega_{1}}^{\omega_{2}} e^{-i t \wp(a)} \frac{f_{a}\left(x^{\prime}\right) f_{-a}(x)}{W(a)} d \wp(a)=\int_{\omega_{2}}^{\omega_{2}+i \omega^{\prime}} e^{-i \wp_{\wp}(b)} \frac{f_{b}(x) f_{-b}\left(x^{\prime}\right)}{W(b)} d \wp(b) .
$$


Hence we have that for $x>x^{\prime}$

$$
\begin{aligned}
K_{1}\left(t, x, x^{\prime}\right) & =\int_{-\wp\left(\omega_{1}\right)}^{-\wp\left(\omega_{2}\right)} e^{i t E}\left(f_{-a}\left(x^{\prime}\right) f_{a}(x)+f_{-a}(x) f_{a}\left(x^{\prime}\right)\right) \frac{d E}{W(E)} \\
& =\int_{\omega_{1}}^{\omega_{1}+i 2 \omega^{\prime}} e^{-i t \wp(a)} \frac{f_{a}(x) f_{-a}\left(x^{\prime}\right)}{W(a)} d(-\wp(a)) \\
& =\int_{\omega_{1}}^{\omega_{1}+i 2 \omega^{\prime}} e^{-i t \wp(a)+i\left(x-x^{\prime}\right) k(a)} m_{a}(x) m_{-a}\left(x^{\prime}\right) \frac{-\wp^{\prime}(a) d a}{W(a)} .
\end{aligned}
$$

Note $k(a)$ is real-valued and by Eq.(2.2), we have that for $x<x^{\prime}$

$$
K_{1}\left(t, x, x^{\prime}\right)=\int_{\omega_{1}}^{\omega_{1}+i 2 \omega^{\prime}} e^{-i t \wp(a)+i\left(x-x^{\prime}\right) k(a)} \overline{m_{a}}\left(x^{\prime}\right) \overline{m_{-a}}(x) \frac{-\wp^{\prime}(a) d a}{\bar{W}(a)} .
$$

To simplify notation, we set $\tau=\frac{x-x^{\prime}}{t} \in \mathbb{R}$ and

$$
F_{\tau}(a)=\wp(a)-i \tau\left(\zeta(a)-\frac{a}{\omega} \zeta(\omega)\right) .
$$

Moreover, we write

$$
K_{1}\left(t, x, x^{\prime}\right)=\int_{\omega_{1}}^{\omega_{1}+i 2 \omega^{\prime}} e^{-i t F_{\tau}(a)} \varphi\left(a, x, x^{\prime}\right) d a
$$

where $\varphi\left(a, x, x^{\prime}\right)=m_{a}(x) m_{-a}\left(x^{\prime}\right) \frac{-\wp^{\prime}(a)}{W(a)}$ when $x>x^{\prime}$, and $\varphi\left(a, x, x^{\prime}\right)=\overline{\varphi\left(a, x^{\prime}, x\right)}$ when $x<x^{\prime}$. Without losing clarity, $\varphi\left(a, x, x^{\prime}\right)$ will be written simply as $\varphi(a)$.

By Remark 2.2, $\varphi\left(a, x, x^{\prime}\right)$ and its $\partial_{a}$-derivatives are bounded uniformly for $a \in\left[\omega_{1}, \omega_{2}\right]$ and $x, x^{\prime} \in \mathbb{R}$. To apply Lemma 2.3 to $(3.3)$, we analyze the $\partial_{a^{-}}$ derivatives of $F_{\tau}(a)$. Our plan is to decompose the integral in (3.3) into several regions and on each region, Lemma 2.3 for some exponent $k$ will be applied. We observe

$$
\begin{aligned}
& \partial_{a} F_{\tau}(a)=\wp^{\prime}(a)+\tau i(\zeta(\omega) / \omega+\wp(a)), \\
& \partial_{a}^{2} F_{\tau}(a)=\wp^{\prime \prime}(a)+\tau i \wp^{\prime}(a), \\
& \partial_{a}^{3} F_{\tau}(a)=\partial_{a}^{3} \wp(a)+\tau i \wp^{\prime \prime}(a) .
\end{aligned}
$$

Let

$$
c_{1}=\min \left\{\zeta(\omega) / \omega+\wp(a): a \in\left[\omega_{1}, \omega_{1}+2 i \omega^{\prime}\right]\right\} .
$$

Then $c_{1}=\zeta(\omega) / \omega+\wp\left(\omega_{2}\right)$ and by Lemma $2.4, c_{1}>0$. Also we denote

$$
M_{1}=1+\max \left\{\left|\wp^{\prime}(a)\right|,\left|\wp^{\prime \prime}(a)\right|, \zeta(\omega) / \omega+\wp(a): a \in\left[\omega_{1}, \omega_{1}+2 i \omega^{\prime}\right]\right\} .
$$

When $|\tau|>\frac{2 M_{1}}{c_{1}}$, we have for $a \in\left[\omega_{1}, \omega_{1}+2 i \omega^{\prime}\right]$

and

$$
\left|\partial_{a} F_{\tau}(a)\right|>|\tau| c_{1}-M_{1}>\frac{1}{2}|\tau| c_{1},
$$

$$
\left|\partial_{a}^{2} F_{\tau}(a)\right|<M_{1}(|\tau|+1)
$$


Integrating by parts and recalling $\varphi(a)$ and its derivatives are uniformly bounded, we obtain

$$
\begin{aligned}
\left|K_{1}\left(t, x, x^{\prime}\right)\right| & =\frac{1}{t}\left|\int_{\omega_{1}}^{\omega_{1}+i 2 \omega^{\prime}} \frac{\varphi(a)}{\partial_{a} F_{\tau}(a)} d e^{-i t F_{\tau}(a)}\right| \\
(3.8) & \leq \frac{4\|\varphi(a)\|_{L^{\infty}\left[\omega_{1}, \omega_{2}\right]}}{t|\tau| c_{1}}+\frac{1}{t}\left|\int_{\omega_{1}}^{\omega_{1}+i 2 \omega^{\prime}} e^{-i t F_{\tau}(a)}\left(\frac{\varphi^{\prime}(a)}{F_{\tau}^{\prime}(a)}-\frac{\varphi(a) F_{\tau}^{\prime \prime}(a)}{\left(F_{\tau}^{\prime}(a)\right)^{2}}\right) d a\right| \\
& \leq C t^{-1} .
\end{aligned}
$$

We now estimate $K_{1}\left(t, x, x^{\prime}\right)$ when $|\tau| \leq \frac{2 M_{1}}{c_{1}}$. Suppose both (3.4) and (3.5) vanish for $a=a_{0} \in\left[\omega_{1}, \omega_{2}\right]$ and $\tau=\tau_{0} \in\left[-\frac{2 M_{1}}{c_{1}}, \frac{2 M_{1}}{c_{1}}\right]$. Then

$$
\wp^{\prime}\left(a_{0}\right)^{2}=\wp^{\prime \prime}\left(a_{0}\right)\left(\frac{\zeta(\omega)}{\omega}+\wp\left(a_{0}\right)\right) .
$$

By Eq.(1.5) and (1.6), this is equivalent to

$$
2 \wp\left(a_{0}\right)^{3}+\frac{6 \zeta(\omega)}{\omega} \wp\left(a_{0}\right)^{2}+\frac{g_{2}}{2} \wp\left(a_{0}\right)+g_{3}-\frac{g_{2} \zeta(\omega)}{2 \omega}=0 .
$$

Thus $\wp\left(a_{0}\right)$ is the simple root of $P(x)$ in $\left[\wp\left(\omega_{2}\right), \wp\left(\omega_{1}\right)\right]$ and $\wp^{\prime}\left(a_{0}\right) \neq 0$ by Lemma 2.4 .

Observe that (3.4) and (3.5) also vanish when $(a, \tau)=\left(2 \omega_{2}-a_{0},-\tau_{0}\right)$. The analysis of $\left(2 \omega_{2}-a_{0},-\tau_{0}\right)$ is the same as that of $\left(a_{0}, \tau_{0}\right)$ and we will focus on $\left(a_{0}, \tau_{0}\right)$. Also we observe that $\partial_{a}^{3} F_{\tau}(a)$ vanishes at $\left(a_{0}, \tau_{0}\right)$ if and only if

$$
\operatorname{det}\left(\begin{array}{cc}
\wp^{\prime}\left(a_{0}\right) & \frac{\zeta(\omega)}{\omega}+\wp\left(a_{0}\right) \\
\partial_{a}^{3} \wp\left(a_{0}\right) & \wp^{\prime \prime}\left(a_{0}\right)
\end{array}\right)=0 ;
$$

namely,

$$
\partial_{a} \operatorname{det}\left(\begin{array}{cc}
\wp^{\prime}(a) & \frac{\zeta(\omega)}{\omega}+\wp(a) \\
\wp^{\prime \prime}(a) & \wp^{\prime}(a)
\end{array}\right)_{a=a_{0}}=0 .
$$

Since $\wp^{\prime}\left(a_{0}\right) \neq 0$, that $\partial_{a}^{3} F_{\tau_{0}}\left(a_{0}\right)=0$ is equivalent to the fact that $\wp\left(a_{0}\right)$ is a double root of $P(x)$. By Lemma 2.4, $P(x)$ has no double root in $\left[\wp\left(\omega_{2}\right), \wp\left(\omega_{1}\right)\right]$. Hence, $\partial_{a}^{3} F_{\tau_{0}}\left(a_{0}\right) \neq 0$ and there exists $\epsilon>0$ such that

$$
\min \left\{\Sigma_{j=1}^{3}\left|\partial_{a}^{j} F_{\tau}(a)\right|: a \in\left[\omega_{1}, \omega_{1}+2 i \omega^{\prime}\right], \tau \in\left[-2 M_{1} / c_{1}, 2 M_{1} / c_{1}\right]\right\}>\epsilon>0 .
$$

Let $\chi_{3}(a, \tau)$ be a smooth function defined on $\left[\omega_{1}, \omega_{1}+2 i \omega^{\prime}\right] \times\left[-\frac{2 M_{1}}{c_{1}}, \frac{2 M_{1}}{c_{1}}\right]$ such that $0 \leq \chi_{3} \leq 1, \chi_{3}(a, \tau)=1$ when $\left|\partial_{a} F_{\tau}(a)\right|+\left|\partial_{a}^{2} F_{\tau}(a)\right| \leq \frac{1}{3} \epsilon$, and $\chi_{3}(a, \tau)=0$ when $\left|\partial_{a} F_{\tau}(a)\right|+\left|\partial_{a}^{2} F_{\tau}(a)\right| \geq \frac{2}{3} \epsilon$. Similarly, let $\chi_{2}(a, \tau)$ to be a smooth function defined on $\left[\omega_{1}, \omega_{1}+2 i \omega^{\prime}\right] \times\left[-\frac{2 M_{1}}{c_{1}}, \frac{2 M_{1}}{c_{1}}\right]$, such that $0 \leq \chi_{2} \leq 1, \chi_{2}(a, \tau)=1$ when $\left|\partial_{a}^{2} F_{\tau}(a)\right| \geq \frac{1}{6} \epsilon$, and $\chi_{2}(a, \tau)=0$ when $\left|\partial_{a}^{2} F_{\tau}(a)\right| \leq \frac{1}{9} \epsilon$.

On the support of $\chi_{3},\left|\partial_{a}^{3} F_{\tau}(a)\right| \geq \frac{1}{3} \epsilon$. It follows from Lemma 2.3 that

$$
\left|\int_{\omega_{1}}^{\omega_{1}+i 2 \omega^{\prime}} e^{-i t F_{\tau}(a)} \chi_{3}(a, \tau) \varphi(a) d a\right| \leq C_{3}(\tau) t^{-\frac{1}{3}} .
$$

On the support of $\chi_{2}\left(1-\chi_{3}\right),\left|\partial_{a}^{2} F_{\tau}(a)\right| \geq \frac{1}{9} \epsilon$. And similarly 


$$
\left|\int_{\omega_{1}}^{\omega_{1}+i 2 \omega^{\prime}} e^{-i t F_{\tau}(a)} \chi_{2}(a, \tau)\left(1-\chi_{3}(a, \tau)\right) \varphi(a) d a\right| \leq C_{2}(\tau) t^{-\frac{1}{2}} .
$$

On the support of $\left(1-\chi_{2}\right)\left(1-\chi_{3}\right),\left|\partial_{a}^{2} F_{\tau}(a)\right| \leq \frac{1}{6} \epsilon$ and $\left|\partial_{a} F_{\tau}(a)\right| \geq \frac{1}{6} \epsilon$. Lemma 2.3 yields

$$
\left|\int_{\omega_{1}}^{\omega_{1}+i 2 \omega^{\prime}} e^{-i t F_{\tau}(a)}\left(1-\chi_{2}(a, \tau)\right)\left(1-\chi_{3}(a, \tau)\right) \varphi(a) d a\right| \leq C_{1}(\tau) t^{-1} .
$$

Note $C_{j}(\tau), j=1,2,3$, are continuous functions of $\tau \in\left[-2 M_{1} / c_{1}, 2 M_{1} / c_{1}\right]$. Let

$$
C=\Sigma_{j=1}^{3} \max \left\{C_{j}(\tau): \tau \in\left[-2 M_{1} / c_{1}, 2 M_{1} / c_{1}\right]\right\} .
$$

Then $\left|K_{1}\left(t, x, x^{\prime}\right)\right| \leq C t^{-\frac{1}{3}}$ for large $t$, because

Consequently, we have proved that for large $t$

$$
\chi_{3}+\chi_{2}\left(1-\chi_{3}\right)+\left(1-\chi_{2}\right)\left(1-\chi_{3}\right)=1 .
$$

$$
\sup _{x, x^{\prime}}\left|K_{1}\left(t, x, x^{\prime}\right)\right|<C t^{-\frac{1}{3}}
$$

where $C$ only depends on $\omega, \omega^{\prime}$.

\section{Analysis of $K_{2}\left(t, x, x^{\prime}\right)$}

We now consider $K_{2}\left(t, x, x^{\prime}\right)$. Let $b=2 \omega_{3}-a$ for $a \in\left(0, \omega_{3}\right)$, and the proof of Lemma 3.1 gives

Then for $x>x^{\prime}$

$$
\frac{f_{a}\left(x^{\prime}\right) f_{-a}(x)}{W(a)}=-\frac{f_{b}(x) f_{-b}\left(x^{\prime}\right)}{W(b)} .
$$

$$
\begin{aligned}
K_{2}\left(t, x, x^{\prime}\right) & =\int_{-\wp\left(\omega_{3}\right)}^{+\infty} e^{i t E}\left(f_{-a}\left(x^{\prime}\right) f_{a}(x)+f_{-a}(x) f_{a}\left(x^{\prime}\right)\right) \frac{d E}{W(E)} \\
& =\int_{-\wp\left(\omega_{3}\right)}^{+\infty} e^{-i t \wp(a)} \frac{f_{a}(x) f_{-a}\left(x^{\prime}\right)}{W(a)} d(-\wp(a)) \\
& =\int_{0}^{i 2 \omega^{\prime}} e^{-i t F_{\tau}(a)} m_{a}(x) m_{-a}\left(x^{\prime}\right) \frac{-\wp^{\prime}(a)}{W(a)} d a
\end{aligned}
$$

where $\tau=\frac{x-x^{\prime}}{t}$. For $x<x^{\prime}, K_{2}\left(t, x, x^{\prime}\right)$ can be written in a similar form. Therefore

$$
K_{2}\left(t, x, x^{\prime}\right)=\int_{0}^{i 2 \omega^{\prime}} e^{-i t F_{\tau}(a)} \varphi\left(a, x, x^{\prime}\right) d a,
$$

where $\varphi\left(a, x, x^{\prime}\right)$ was defined in the previous section.

Step 1. The analysis of the nonlinear phase in $K_{2}\left(t, x, x^{\prime}\right)$ is similar to that of $K_{1}\left(t, x, x^{\prime}\right)$. However, by Remark $2.2, \varphi\left(a, x, x^{\prime}\right)$ in $K_{2}\left(t, x, x^{\prime}\right)$ is unbounded when $a \rightarrow 0$ and $a \rightarrow 2 i \omega^{\prime}$, contrary to the case of $K_{1}\left(t, x, x^{\prime}\right)$. Our strategy then is to change variables to remove this singularity. 
Define $\lambda^{2}=\wp\left(\omega_{3}\right)-\wp(a)$ such that $\lambda>0$ when $a \in\left(0, \omega_{3}\right)$ and $\lambda<0$ when $a \in\left(\omega_{3}, 2 \omega_{3}\right)$. Then the map $a \rightarrow \lambda$ is one-to-one, onto and analytic from $\left(0,2 \omega_{3}\right)$ to $\mathbb{R}$. Note that $\lambda\left(2 i \omega^{\prime}-a\right)=-\lambda(a)$ and the behavior of $\lambda(a)$ as $a \rightarrow 2 i \omega^{\prime}$ is the same as that when $a \rightarrow 0$.

We claim that $\frac{\partial a}{\partial \lambda}=\frac{2 \lambda}{-\wp^{\prime}(a)}$ is never zero when $a \in\left(0,2 \omega_{3}\right)$. In fact, the claim is obvious for $a \neq \omega_{3}$. When $a=\omega_{3}$, by L'Hopital's Rule,

which implies that

$$
\frac{\partial a}{\partial \lambda}(0)=\lim _{\lambda \rightarrow 0} \frac{2 \lambda}{-\wp^{\prime}(a)}=\lim _{\lambda \rightarrow 0} \frac{2}{-\wp^{\prime \prime}(a) \frac{\partial a}{\partial \lambda}},
$$

$$
\left|\frac{\partial a}{\partial \lambda}(0)\right|=\sqrt{\frac{2}{\wp^{\prime \prime}\left(\omega_{3}\right)}}>0 .
$$

Observe that when $\lambda \rightarrow \pm \infty, \lambda \varphi(\lambda)=\lambda^{3} \cdot O(1)$ and $\left|-\wp^{\prime}(a)\right|=|\lambda|^{3}+O\left(\lambda^{2}\right)$. Hence, $\frac{\lambda \varphi(\lambda)}{-\wp^{\prime}(a)}$ and its $\lambda$-derivatives are bounded uniformly for $x, x^{\prime}, \lambda \in \mathbb{R}$.

After changing the variables, we obtain

$$
\int_{0}^{i 2 \omega^{\prime}} e^{-i t F_{\tau}(a)} \varphi(a) d a=\int_{\mathbb{R}} e^{-i t F_{\tau}(\lambda)} \varphi(\lambda) \frac{\partial a}{\partial \lambda} d \lambda,
$$

where $F_{\tau}(\lambda)=F_{\tau}(a(\lambda))$ and $\varphi(\lambda)=\varphi(a(\lambda))$.

We will decompose (4.1) into different integral regions and estimate them separately. Define $\chi(\cdot)$ to be a smooth function supported in $(-2,2)$ such that $\chi(x)=1$ when $x \in[-1,1]$, and let $M$ be a large number to be specified.

Step 2. we claim

$$
\left|\int_{\mathbb{R}} e^{-i t F_{\tau}(\lambda)} \varphi(\lambda) \frac{2 \lambda}{-\wp^{\prime}(a)} \chi(\lambda / M) d \lambda\right|<C_{M} t^{-\frac{1}{4}} \text { or } C_{M} t^{-\frac{1}{3}},
$$

depending on whether $P(x)$ has a double root in $\left(-\infty, \wp\left(\omega_{3}\right)\right]$ or not.

The proof of (4.2) will follow the lines of the proof of (3.12). Recall that the map $\lambda \rightarrow a$ is one-to-one from $\mathbb{R}$ onto $\left(0,2 \omega_{3}\right)$, and satisfies $\lambda^{2}=\wp\left(\omega_{3}\right)-\wp(a)$. Also, we observe

$$
\begin{aligned}
& \partial_{\lambda} F_{\tau}(\lambda)=\frac{\partial a}{\partial \lambda}\left(\wp^{\prime}(a)+\tau i(\zeta(\omega) / \omega+\wp(a))\right), \\
& \partial_{\lambda}^{2} F_{\tau}(\lambda)=\frac{\partial^{2} a}{\partial \lambda^{2}}\left(\wp^{\prime}(a)+\tau i(\zeta(\omega) / \omega+\wp(a))\right)+\left(\frac{\partial a}{\partial \lambda}\right)^{2}\left(\wp^{\prime \prime}(a)+\tau i \wp^{\prime}(a)\right) .
\end{aligned}
$$

By Lemma 2.4,

$$
\inf \left\{|\zeta(\omega) / \omega+\wp(a)|: a \in\left(0, \omega_{3}\right)\right\}=\left|\zeta(\omega) / \omega+\wp\left(\omega_{3}\right)\right|=c_{2}>0 .
$$

Denote

$$
M_{2}=\max \left\{\left|\wp^{\prime}(a)\right|+\left|\wp^{\prime \prime}(a)\right|+|\zeta(\omega) / \omega+\wp(a)|: \lambda(a) \in[-2 M, 2 M]\right\} .
$$

Since $\frac{\partial a}{\partial \lambda}$ is smooth and never zero, there exist $c_{3}$ and $M_{3}$ such that $0<c_{3}<\left|\frac{\partial a}{\partial \lambda}\right|<$ $\sqrt{M_{3}}$ for all $\lambda \in[-2 M, 2 M]$. Moreover, suppose $\left|\frac{\partial^{2} a}{\partial \lambda^{2}}\right|<M_{3}$ for $\lambda \in[-2 M, 2 M]$. Then for $\lambda \in[-2 M, 2 M]$ and $|\tau| \geq 2 M_{2} / c_{2}$ 


$$
\left|\partial_{\lambda} F_{\tau}(\lambda)\right|>\frac{1}{2} c_{2} c_{3}|\tau|, \quad\left|\partial_{\lambda}^{2} F_{\tau}(\lambda)\right|<2 M_{3} M_{2}(1+|\tau|) .
$$

Integrating by parts, an argument similar to (3.8) shows that for $|\tau| \geq 2 M_{2} / c_{2}$

$$
\left|\int_{\mathbb{R}} e^{-i t F_{\tau}(\lambda)} \varphi(\lambda) \frac{2 \lambda}{-\wp^{\prime}(a)} \chi(\lambda / M) d \lambda\right|<C_{M} t^{-1} .
$$

To prove (4.2) for $|\tau| \leq 2 M_{2} / c_{2}$, we first suppose that $P(x)$ has no double root in $\left(-\infty, \wp\left(\omega_{3}\right)\right]$. Since $\frac{\partial F_{\tau}(\lambda)}{\partial \lambda}=\frac{\partial a}{\partial \lambda} \frac{\partial F_{\tau}(a)}{\partial a}$ and $\frac{\partial a}{\partial \lambda} \neq 0$ for $a \in\left(0,2 \omega_{3}\right)$, it follows from (4.3) and (4.4) that $\frac{\partial F_{\tau}(\lambda)}{\partial \lambda}$ and $\frac{\partial^{2} F_{\tau}(\lambda)}{\partial \lambda^{2}}$ vanish at $\left(\lambda_{0}, \tau_{0}\right)$ if and only if $(3.4)$ and (3.5) vanish at $\left(a_{0}, \tau_{0}\right)$, where $\lambda_{0}^{2}=\wp\left(\omega_{3}\right)-\wp\left(a_{0}\right)$. Therefore, the fact that $P(x)$ has no double root implies that there exists $\epsilon$ such that

$$
\min \left\{\sum_{j=1}^{3}\left|\partial_{\lambda}^{j} F_{\tau}(\lambda)\right|:|\lambda| \leq 2 \lambda|\tau|<2 M_{2} / c_{2}\right\}>\epsilon>0 .
$$

Just as in the case of $K_{1}\left(t, x, x^{\prime}\right)$, we define $\chi_{2}(\lambda, \tau), \chi_{3}(\lambda, \tau)$ on $[-2 M, 2 M] \times$ $\left[-2 M_{2} / c_{2}, 2 M_{2} / c_{2}\right]$. Namely, $\chi_{2}(\lambda, \tau)=1$ when $\left|\partial_{\lambda}^{2} F_{\tau}(\lambda)\right|>\frac{1}{6} \epsilon$, and $\chi_{2}(\lambda, \tau)=0$ when $\left|\partial_{\lambda}^{2} F_{\tau}(\lambda)\right|<\frac{1}{9} \epsilon$. $\chi_{3}(\lambda, \tau)=1$ when $\left|\partial_{\lambda} F_{\tau}(\lambda)\right|+\left|\partial_{\lambda}^{2} F_{\tau}(\lambda)\right|<\frac{1}{3} \epsilon$, and $\chi_{3}(\lambda, \tau)=$ 0 when $\left|\partial_{\lambda} F_{\tau}(\lambda)\right|+\left|\partial_{\lambda}^{2} F_{\tau}(\lambda)\right|>\frac{2}{3} \epsilon$.

Decompose the integral in (4.2) according to

$$
1=\chi_{3}+\chi_{2}\left(1-\chi_{3}\right)+\left(1-\chi_{2}\right)\left(1-\chi_{3}\right) \text {. }
$$

The same arguments as that in (3.9), (3.10) and (3.11) yield for $|\tau| \leq 2 M_{2} / c_{2}$

$$
\left|\int_{\mathbb{R}} e^{-i t F_{\tau}(\lambda)} \varphi(\lambda) \frac{2 \lambda}{-\wp^{\prime}(a)} \chi(\lambda / M) d \lambda\right|<C_{M} t^{-\frac{1}{3}} .
$$

In the case that $P(x)$ has a double root $\wp\left(a_{0}\right) \in\left(-\infty, \wp\left(\omega_{3}\right)\right]$, there is $\tau_{0} \in \mathbb{R}$, such that $\partial_{\lambda}^{j} F_{\tau}(\lambda), j=1,2,3$ vanish at $\left(\lambda_{0}, \tau_{0}\right)$, where $\lambda_{0}^{2}=\wp\left(\omega_{3}\right)-\wp\left(a_{0}\right)$. The fact that $P(x)$ has no root of degree 3 implies that $\partial_{\lambda}^{4} F_{\tau_{0}}\left(\lambda_{0}\right) \neq 0$. Therefore, there exists $\epsilon$ such that

$$
\min \left\{\sum_{j=1}^{4}\left|\partial_{\lambda}^{j} F_{\tau}(\lambda)\right|: \lambda \in[-2 M, 2 M], \tau \in\left[-2 M_{2} / c_{2}, 2 M_{2} / c_{2}\right]\right\}>2 \epsilon>0
$$

Define smooth function $\chi_{4}(\lambda, \tau):[-2 M, 2 M] \times\left[-2 M_{2} / c_{2}, 2 M_{2} / c_{2}\right] \rightarrow[0,1]$, such that $\chi_{4}=1$ when $\Sigma_{j=1}^{3}\left|\partial_{\lambda}^{j} F_{\tau}(\lambda)\right| \leq \epsilon$, and $\chi_{4}=0$ when $\Sigma_{j=1}^{3}\left|\partial_{\lambda}^{j} F_{\tau}(\lambda)\right| \geq \frac{3}{2} \epsilon$. Hence on the support of $\chi_{4},\left|\partial_{\lambda}^{4} F_{\tau}(\lambda)\right| \geq \frac{1}{2} \epsilon$. It follows from Lemma 2.3 that

$$
\left|\int_{\mathbb{R}} e^{-i t F_{\tau}(\lambda)} \varphi(\lambda) \frac{2 \lambda}{-\wp^{\prime}(a)} \chi(\lambda / M) \chi_{4}(\lambda, \tau) d \lambda\right|<C_{4}(\tau) t^{-\frac{1}{4}} .
$$

We decompose the integral in (4.2) by using

$$
\chi_{4}+\left(1-\chi_{4}\right) \chi_{3}+\chi_{2}\left(1-\chi_{3}\right)\left(1-\chi_{4}\right)+\left(1-\chi_{2}\right)\left(1-\chi_{3}\right)\left(1-\chi_{4}\right)=1 .
$$

The analysis of the terms containing $\left(1-\chi_{4}\right) \chi_{3}, \chi_{2}\left(1-\chi_{3}\right)\left(1-\chi_{4}\right)$ and $(1-$ $\left.\chi_{2}\right)\left(1-\chi_{3}\right)\left(1-\chi_{4}\right)$ is similar to (3.9), (3.10) and (3.11) respectively. Therefore, under the assumption that $P(x)$ has a double root in $\left(-\infty, \wp\left(\omega_{3}\right)\right]$, we have proved 


$$
\left|\int_{\mathbb{R}} e^{-i t F_{\tau}(\lambda)} \varphi(\lambda) \frac{2 \lambda}{-\wp^{\prime}(a)} \chi(\lambda / M) d \lambda\right|<C_{M} t^{-\frac{1}{4}}
$$

Step 3. It now remains to estimate

which by definition equals

$$
\int_{\mathbb{R}} e^{-i t F_{\tau}(\lambda)} \varphi(\lambda) \frac{2 \lambda}{-\wp^{\prime}(a)}(1-\chi(\lambda / M)) d \lambda,
$$

$$
\lim _{N \rightarrow+\infty} \int_{\mathbb{R}} e^{-i t F_{\tau}(\lambda)} \varphi(\lambda) \frac{2 \lambda}{-\wp^{\prime}(a)}(\chi(\lambda / N)-\chi(\lambda / M)) d \lambda .
$$

Since $\frac{2 \lambda \varphi(\lambda)}{-\wp^{\prime}(a)}$ are not integrable on the support of $1-\chi(\lambda / M)$, Lemma 2.3 cannot be applied to (4.5) directly. We shall explore the oscillation of the phase $e^{-i t F_{\tau}(\lambda)}$ and perform integration by parts to bound (4.5), which requires us to exclude the zeroes of $\partial_{\lambda} F_{\tau}(\lambda)$.

By definition, $\wp(a)=a^{-2}+\frac{1}{20} g_{2} a^{2}+O\left(a^{4}\right)$, and $\wp(a)=\wp\left(i \omega^{\prime}\right)-\lambda^{2}$, hence

$$
\lambda(a)=\frac{i}{a}+\alpha_{1} a+O\left(a^{3}\right) \quad \text { as } a \rightarrow 0, a \in\left(0, \omega_{3}\right)
$$

which is an meromorphic function of $a$. It follows that $\zeta(a)=-i \lambda+O\left(\lambda^{-1}\right)$ as $a \rightarrow 0, a \in\left(0, \omega_{3}\right)$. Consequently

$$
\begin{aligned}
F_{\tau}(\lambda) & =-\lambda^{2}-\tau \lambda+\wp\left(i \omega^{\prime}\right)+O\left(\frac{\tau}{\lambda}\right), \quad \lambda \rightarrow \pm \infty \\
\partial_{\lambda} F_{\tau}(\lambda) & =-2 \lambda-\tau+O\left(\tau \lambda^{-2}\right), \quad \lambda \rightarrow \pm \infty \\
\partial_{\lambda}^{2} F_{\tau}(\lambda) & =-2+O\left(\tau \lambda^{-3}\right), \quad \lambda \rightarrow \pm \infty
\end{aligned}
$$

We require $M$ large enough such that

$$
M>1+\max \left\{\left|\lambda_{0}\right|: \frac{\partial F_{\tau}(\lambda)}{\partial \lambda}, \frac{\partial^{2} F_{\tau}(\lambda)}{\partial \lambda^{2}} \text { both vanish at }\left(\tau_{0}, \lambda_{0}\right)\right\} .
$$

Therefore, if $|\lambda|>M, \frac{\partial F_{\tau}(\lambda)}{\partial \lambda}$ and $\frac{\partial^{2} F_{\tau}(\lambda)}{\partial \lambda^{2}}$ cannot vanish at the same $(\tau, \lambda)$.

When $|\lambda|>M$ and $\left|\lambda+\frac{\tau}{2}\right|>1$, we claim

$$
\left|\partial_{\lambda} F_{\tau}(\lambda)\right|>\left|\lambda+\frac{\tau}{2}\right|-\frac{1}{2}
$$

In fact, by (4.7)

$$
\left|\partial_{\lambda} F_{\tau}(\lambda)\right|>2\left|\lambda+\frac{\tau}{2}\right|-O\left(\tau \lambda^{-2}\right)
$$

We choose $M$ large enough such that $O\left(\tau \lambda^{-2}\right)<\frac{|\tau|}{100|\lambda|}$. If $\left|\lambda+\frac{\tau}{2}\right|>\frac{|\tau|}{100}$, (4.9) clearly holds. If $\left|\lambda+\frac{\tau}{2}\right| \leq \frac{|\tau|}{100}$, then $\frac{|\tau|}{100|\lambda|}<\frac{1}{2}$ and (4.9) also follows.

When $\left(-\frac{\tau}{2}-1,-\frac{\tau}{2}+1\right)$ is not contained in $(-M, M)$, by $(4.8),\left|\partial_{\lambda}^{2} F_{\tau}(\lambda)\right|>1$ for $\lambda \in\left(-\frac{\tau}{2}-2,-\frac{\tau}{2}+2\right)$ as long as $M$ is large enough. By Lemma 2.3, we have

$$
\int_{\mathbb{R}} e^{-i t F_{\tau}(\lambda)} \varphi(\lambda) \frac{2 \lambda}{-\wp^{\prime}(a)} \chi(\lambda+\tau / 2) d \lambda<C t^{-\frac{1}{2}},
$$

where $\chi(x)=1$ when $|x|<1$, and $\chi(x)=0$ when $|x|>2$. 
To estimate (4.5), we first consider the case when $\frac{|\tau|}{10}>M$ and estimate

which equals

$$
\int_{\mathbb{R}} e^{-i t F_{\tau}(\lambda)} \frac{2 \lambda \varphi(\lambda)}{-\wp^{\prime}(a)}(\chi(10 \lambda /|\tau|)-\chi(\lambda / M)) d \lambda
$$

$$
\frac{1}{i t} \int_{\mathbb{R}} e^{-i t F_{\tau}(\lambda)} \frac{d}{d \lambda}\left(\frac{2 \lambda \varphi(\lambda)}{-\wp^{\prime}(a)} \frac{\chi(10 \lambda /|\tau|)-\chi(\lambda / M)}{\partial_{\lambda} F(\lambda)}\right) d \lambda .
$$

It follows from Eq.(4.7) that on the support of $\chi(10 \lambda /|\tau|)-\chi(\lambda / M)$

$$
\left|\partial_{\lambda} F_{\tau}(\lambda)\right|>|\tau|-2|\lambda|-O\left(\tau \lambda^{-2}\right)>|\tau| / 2,
$$

and

$$
\left|\partial_{\lambda}^{2} F_{\tau}(\lambda)\right|<|\tau| / 4
$$

as long as $M$ is large enough. Hence

$$
\left|\partial_{\lambda}\left(\partial_{\lambda} F_{\tau}(\lambda)\right)^{-1}\right|<\frac{1}{|\tau|}
$$

Since $(\chi(10 \lambda /|\tau|)-\chi(\lambda / M)), \frac{2 \lambda \varphi(\lambda)}{-\wp^{\prime}(a)}$ and their $\lambda$-derivatives are uniformly bounded, we have

$$
\left|\frac{d}{d \lambda}\left(\frac{2 \lambda \varphi(\lambda)}{-\wp^{\prime}(a)} \frac{\chi(10 \lambda /|\tau|)-\chi(\lambda / M)}{\partial_{\lambda} F(\lambda)}\right)\right|<\frac{C}{|\tau|},
$$

from which it follows that $|(4.10)|<C t^{-1}$.

To complete the estimate on (4.5) when $|\tau| / 10>M$, it remains to bound

$$
\int_{\mathbb{R}} e^{-i t F_{\tau}(\lambda)} \varphi(\lambda) \frac{2 \lambda}{-\wp^{\prime}(a)}(\chi(\lambda / N)-\chi(10 \lambda /|\tau|)-\chi(\lambda+\tau / 2)) d \lambda
$$

Integrating by parts, this equals

$$
\frac{2}{i t} \int_{\mathbb{R}} e^{-i t F_{\tau}(\lambda)} \frac{d}{d \lambda}\left(\frac{\lambda \varphi(\lambda)}{-\wp^{\prime}(a)} \frac{(\chi(\lambda / N)-\chi(10 \lambda / \tau)-\chi(\lambda+\tau / 2))}{\partial_{\lambda} F_{\tau}(\lambda)}\right) d \lambda:=J_{1}+J_{2},
$$

where

$$
J_{1}=\frac{2}{i t} \int_{\mathbb{R}} e^{-i t F_{\tau}(\lambda)} \frac{(\chi(\lambda / N)-\chi(10 \lambda /|\tau|)-\chi(\lambda+\tau / 2))}{\partial_{\lambda} F_{\tau}(\lambda)} \frac{d}{d \lambda} \frac{\lambda \varphi(\lambda)}{-\wp^{\prime}(a)} d \lambda,
$$

and

$$
J_{2}=\frac{2}{i t} \int_{\mathbb{R}} e^{-i t F_{\tau}(\lambda)} \frac{\lambda \varphi(\lambda)}{-\wp^{\prime}(a)} \frac{d}{d \lambda} \frac{(\chi(\lambda / N)-\chi(10 \lambda /|\tau|)-\chi(\lambda+\tau / 2))}{\partial_{\lambda} F_{\tau}(\lambda)} d \lambda .
$$

In $J_{2}$,

$$
\partial_{\lambda}(\chi(\lambda / N)-\chi(10 \lambda /|\tau|)-\chi(\lambda+\tau / 2))=\frac{1}{N} \chi^{\prime}(\lambda / N)-\frac{10}{|\tau|} \chi^{\prime}(10 \lambda /|\tau|)-\chi^{\prime}(\lambda+\tau / 2) .
$$

On the support of (4.12), we have $|\lambda+\tau / 2|>1$ and $|\lambda|>M$. Thus $\left|\left(\partial_{\lambda} F_{\tau}(\lambda)\right)^{-1}\right|<$ $C$ by (4.9). Consequently,

$$
\left|\frac{2}{i t} \int_{\mathbb{R}} e^{-i t F_{\tau}(\lambda)} \frac{\lambda \varphi(\lambda)}{-\wp^{\prime}(a) \partial_{\lambda} F_{\tau}(\lambda)}\left(\frac{1}{N} \chi^{\prime}(\lambda / N)-\frac{10}{|\tau|} \chi^{\prime}(10 \lambda /|\tau|)-\chi^{\prime}(\lambda+\tau / 2)\right) d \lambda\right|<C t^{-1} .
$$


On the support of $\chi(\lambda / N)-\chi(10 \lambda /|\tau|)-\chi(\lambda+\tau / 2)$ we have $|\lambda|>|\tau / 10|$. It follows from (4.8) that $\left|\partial_{\lambda}^{2} F_{\tau}(\lambda)\right|<3$. Combining it with (4.9), we obtain

$$
\partial_{\lambda}\left(\partial_{\lambda} F_{\tau}(\lambda)\right)^{-1}<C\left(\lambda+\frac{\tau}{2}\right)^{-2}
$$

which is integrable. Therefore

$$
\left|\frac{2}{i t} \int_{\mathbb{R}} e^{-i t F_{\tau}(\lambda)} \frac{\lambda \varphi(\lambda)}{-\wp^{\prime}(a)}\left(\partial_{\lambda} \frac{1}{\partial_{\lambda} F_{\tau}(\lambda)}\right)(\chi(\lambda / N)-\chi(10 \lambda /|\tau|)-\chi(\lambda+\tau / 2)) d \lambda\right|<C t^{-1} .
$$

This completes the estimate on $J_{2}$.

As for $J_{1}$, integrating by parts again, we obtain

$$
J_{1}=-\frac{4}{t^{2}} \int_{\mathbb{R}} e^{-i t F_{\tau}(\lambda)} \frac{d}{d \lambda}\left(\frac{(\chi(\lambda / N)-\chi(10 \lambda /|\tau|)-\chi(\lambda+\tau / 2))}{\left(\partial_{\lambda} F_{\tau}(\lambda)\right)^{2}} \frac{d}{d \lambda} \frac{\lambda \varphi(\lambda)}{-\wp^{\prime}(a)}\right) d \lambda .
$$

Applying the Leibnitz's rule, we are left with three terms. Two terms come from $\frac{d}{d \lambda}$ hitting $\chi(\lambda / N)-\chi(10 \lambda /|\tau|)-\chi(\lambda+\tau / 2)$ and $\left(\partial_{\lambda} F_{\tau}(\lambda)\right)^{-2}$, and the analysis is analogous to that of $J_{2}$. When $\frac{d}{d \lambda}$ hits $\frac{d}{d \lambda} \frac{\lambda \varphi(\lambda)}{-\wp^{\prime}(a)}$, we obtain the third term

$$
-\frac{4}{t^{2}} \int_{\mathbb{R}} e^{-i t F_{\tau}(\lambda)} \frac{\chi(\lambda / N)-\chi(10 \lambda /|\tau|)-\chi(\lambda+\tau / 2)}{\left(\partial_{\lambda} F_{\tau}(\lambda)\right)^{2}} \frac{d^{2}}{d \lambda^{2}} \frac{\lambda \varphi(\lambda)}{-\wp^{\prime}(a)} d \lambda
$$

Because $\left|\left(\partial_{\lambda} F_{\tau}(\lambda)\right)^{2}\right|>|\lambda+\tau / 2|^{2} / 4$ on the support of $\chi(\lambda / N)-\chi(10 \lambda /|\tau|)-\chi(\lambda+$ $\tau / 2)$ and $\frac{d^{2}}{d \lambda^{2}} \frac{\lambda \varphi(\lambda)}{-\wp^{\prime}(a)}$ is uniformly bounded, the above term is dominated by $C t^{-2}$, where the constant $C$ is independent of $N$.

This completes the estimate of (4.5) when $|\tau| / 10>M$. The analysis is similar and even simpler when $|\tau| / 10 \leq M$. Therefore, when $P(x)$ has no double root in $\left(-\infty, \wp\left(\omega_{3}\right)\right]$, we have

$$
\sup _{x, x^{\prime}}\left|K_{2}\left(t, x, x^{\prime}\right)\right|<C t^{-\frac{1}{3}} .
$$

The decay factor $t^{-\frac{1}{3}}$ is replaced by $t^{-\frac{1}{4}}$ when $P(x)$ has a double root in $\left(-\infty, \wp\left(\omega_{3}\right)\right]$.

Combining the estimates on $K_{1}\left(t, x, x^{\prime}\right)$ and $K_{2}\left(t, x, x^{\prime}\right)$, we have proved (1.11) under the assumption (1.15). We have also proved (1.12) for all nonzero $\omega, \omega^{\prime} \in \mathbb{R}$.

It remains to prove (1.15) holds for almost all $\omega, \omega^{\prime} \in \mathbb{R}$. Recall

Suppose $P(x)$ has a double root $x_{0} \in\left(-\infty, \wp\left(\omega_{3}\right)\right]$. Then $x_{0}$ is a root of $P^{\prime}(x)$.

with its roots

$$
P^{\prime}(x)=6 x^{2}+\frac{12 \zeta(\omega)}{\omega} x+\frac{g_{2}}{2}
$$

$$
r_{+}, r_{-}=-\frac{\zeta(\omega)}{\omega} \pm \sqrt{\left(\frac{\zeta(\omega)}{\omega}\right)^{2}-\frac{g_{2}}{12}}
$$

That $P(x)$ has a double root in $\left(-\infty, \wp\left(\omega_{3}\right)\right]$ implies that $(\zeta(\omega) / \omega)^{2}-g_{2} / 12>0$ and $P\left(r_{-}\right)=0$. By $(6.1), g_{2}$ and $g_{3}$ are real analytic for $\omega, \omega^{\prime} \in \mathbb{R}^{+}$. By $(6.3), \zeta(\omega)$ is also real analytic for $\omega, \omega^{\prime} \in \mathbb{R}^{+}$. Therefore, $r_{+}, r_{-}$are analytic when $\omega, \omega^{\prime} \in \mathbb{R}^{+}$, with branches at $\left(\frac{\zeta(\omega)}{\omega}\right)^{2}-\frac{g_{2}}{12}=0$. To prove $(1.15)$ is true for almost all $\omega, \omega^{\prime} \in \mathbb{R}^{+}$, 
it suffices to show that $P\left(r_{-}\right)$is nonzero at one point. This can be done by direct numerical calculation.

For example, take $\omega=5.5$ and $\omega^{\prime}=2$. Then we have

$$
\begin{gathered}
g_{2}=0.507343, \quad g_{3}=-0.0695438, \\
r_{+}, r_{-}=0.0628169 \pm 0.195787 i, \\
P\left(r_{+}\right), P\left(r_{-}\right)=-0.0386656 \pm 0.0300201 i .
\end{gathered}
$$

This indicates that $P\left(r_{-}\right)$is nonzero for almost all $\omega, \omega^{\prime} \in \mathbb{R}$. Therefore, (1.15) holds for almost all $\omega, \omega^{\prime} \in \mathbb{R}$.

\section{Optimality of the decay factor}

So far we have proved the first part of Theorem 1.1. To verify (1.13), we first reduce it to showing that there exist constants $c>0$ and $T>0$ such that for $t>T$

$$
\left\|K\left(t, x, x^{\prime}\right)\right\|_{L^{\infty}}>c t^{-\frac{1}{3}} .
$$

Accepting (5.1) temporarily, we obtain that for any given large $t$, there exist $\left(x_{0}, x_{0}^{\prime}\right)$ such that $x_{0} \neq x_{0}^{\prime}$ and $\left|K\left(t, x_{0}, x_{0}^{\prime}\right)\right|>c t^{-\frac{1}{3}}$. Without loss of generality, suppose that

$$
\Re\left(K\left(t, x_{0}, x_{0}^{\prime}\right)\right)>\frac{c}{2} t^{-\frac{1}{3}}
$$

As $K\left(t, x, x^{\prime}\right)$ is smooth away from $x=x^{\prime}$, there exists $\delta>0$ such that for any $\left(x, x^{\prime}\right) \in\left(x_{0}-\delta, x_{0}+\delta\right) \times\left(x_{0}^{\prime}-\delta, x_{0}^{\prime}+\delta\right)$

$$
\Re\left(K\left(t, x, x^{\prime}\right)\right)>\frac{c}{4} t^{-\frac{1}{3}} .
$$

Take the initial data $\psi_{0}\left(x^{\prime}\right)=\frac{1}{2 \delta} \chi_{\left(x_{0}^{\prime}-\delta, x_{0}^{\prime}+\delta\right)}\left(x^{\prime}\right)$. Then $\left\|\psi_{0}\right\|_{L^{1}}=1$ and for any $x \in\left(x_{0}-\delta, x_{0}+\delta\right)$

$$
|\psi(t, x)|=\left|\int K\left(t, x, x^{\prime}\right) \psi_{0}\left(x^{\prime}\right) d x^{\prime}\right|>\frac{c}{4} t^{-\frac{1}{3}} .
$$

To prove (5.1), we need the following lemma (Prop.3 Chap.8 [St]):

Lemma 5.1. Suppose $k \geq 2$, and

$$
\phi\left(x_{0}\right)=\phi^{\prime}\left(x_{0}\right)=\cdots=\phi^{(k-1)}\left(x_{0}\right)=0,
$$

while $\phi^{(k)}\left(x_{0}\right) \neq 0$. If $\psi$ is supported on a sufficiently small neighborhood of $x_{0}$ and $\psi\left(x_{0}\right) \neq 0$, then

$$
\int_{\mathbb{R}} e^{i \lambda \phi(x)} \psi(x) d x=a_{k} \psi\left(x_{0}\right)\left(\phi^{(k)}\left(x_{0}\right)\right)^{-\frac{1}{k}} \lambda^{-\frac{1}{k}}+O\left(\lambda^{-\frac{1}{k}-1}\right),
$$

where $a_{k} \neq 0$ only depends on $k$. The implicit constant in $O\left(\lambda^{-\frac{1}{k}-1}\right)$ depends on only finitely many derivatives of $\phi$ and $\psi$ at $x_{0}$. 
By Lemma 2.4, $P(a)$ has a unique simple root in $\left(\wp\left(\omega_{2}\right), \wp\left(\omega_{1}\right)\right)$, thus we can choose $a_{0} \in\left(\omega_{1}, \omega_{2}\right)$ and a corresponding $\tau_{0}$ such that both $\partial_{a} F_{\tau}(a)$ and $\partial_{a}^{2} F_{\tau}(a)$ vanish at $\left(a_{0}, \tau_{0}\right)$.

First, we denote $I=\left[0, i 2 \omega^{\prime}\right] \cup\left[\omega_{1}, \omega_{1}+i 2 \omega^{\prime}\right]$ and assume that for any $a \in I$, $a \neq a_{0}$, at least one of $\partial_{a} F_{\tau_{0}}(a)$ and $\partial_{a}^{2} F_{\tau_{0}}(a)$ does not vanish. Then we take $\delta>0$ small enough such that for $a \notin\left(a_{0}-\delta, a_{0}+\delta\right) \subset I,\left|\partial_{a} F_{\tau_{0}}(a)\right|+\left|\partial_{a}^{2} F_{\tau_{0}}(a)\right|$ is greater than some positive constant.

Given any large $t$, take $\left(x, x^{\prime}\right)$ such that $\frac{x-x^{\prime}}{t}=\tau_{0}$ and

$$
K\left(t, x, x^{\prime}\right)=\left(\int_{0}^{i 2 \omega^{\prime}}+\int_{\omega_{1}}^{\omega_{1}+i 2 \omega^{\prime}}\right) e^{-i t F_{\tau_{0}}(a)} m_{a}(x) m_{-a}\left(x^{\prime}\right) \frac{-\wp^{\prime}(a) d a}{W(a)} .
$$

The $\int_{0}^{i 2 \omega^{\prime}}$-term is bounded by $C t^{-\frac{1}{2}}$ using an argument analogous to that in Section 4 , because $\left|\partial_{a} F_{\tau_{0}}(a)\right|+\left|\partial_{a}^{2} F_{\tau_{0}}(a)\right|$ is uniformly greater than some positive constant for $a \in\left(0, i 2 \omega^{\prime}\right)$.

We decompose the $\int_{\omega_{1}}^{\omega_{1}+i 2 \omega^{\prime}}$-term as follows

where

$$
\int_{\omega_{1}}^{\omega_{1}+i 2 \omega^{\prime}} e^{-i t F_{\tau_{0}}(a)} m_{a}(x) m_{-a}\left(x^{\prime}\right) \frac{-\wp^{\prime}(a) d a}{W(a)}:=J_{3}+J_{4},
$$

and

$$
J_{3}=\int_{\omega_{1}}^{\omega_{1}+i 2 \omega^{\prime}} e^{-i t F_{\tau_{0}}(a)} \rho(a) m_{a}(x) m_{-a}\left(x^{\prime}\right) \frac{-\wp^{\prime}(a) d a}{W(a)},
$$

$$
J_{4}=\int_{\omega_{1}}^{\omega_{1}+i 2 \omega^{\prime}} e^{-i t F_{\tau_{0}}(a)} \tilde{\rho}(a) m_{a}(x) m_{-a}\left(x^{\prime}\right) \frac{-\wp^{\prime}(a) d a}{W(a)} .
$$

Here $\rho(a)$ is a smooth cut-off function supported on $\left(a_{0}-\delta, a_{0}+\delta\right)$ and $\tilde{\rho}(a)=$ $1-\rho(a)$.

Under our assumption, $\left|J_{4}\right|<C t^{-\frac{1}{2}}$, following the same reasoning as that in Section 3.

Considering $J_{3}$, the phase function $F_{\tau_{0}}(a)$ satisfies $\partial_{a} F_{\tau_{0}}\left(a_{0}\right)=\partial_{a}^{2} F_{\tau_{0}}\left(a_{0}\right)=0$ and $\partial_{a}^{3} F_{\tau_{0}}\left(a_{0}\right) \neq 0$. $m_{a}(x)$ and $m_{-a}\left(x^{\prime}\right)$ do not vanish when $a \in\left(\omega_{1}, \omega_{2}\right)$ by Eq.(1.9). $\frac{-\wp^{\prime}(a)}{W(a)}$ is nonzero when $a \in\left(\omega_{1}, \omega_{2}\right)$. Therefore $m_{a_{0}}(x) m_{-a_{0}}\left(x^{\prime}\right) \frac{-\wp^{\prime}\left(a_{0}\right)}{W\left(a_{0}\right)}$ is nonzero.

Since $\rho(a)$ is supported in a sufficiently small neighborhood of $a_{0}$, by Lemma 5.1, there exist $c_{1}>0$ and $T>0$ such that for $t>T$

$$
\left|J_{3}\right|>c_{1} t^{-\frac{1}{3}}
$$

where $c_{1}$ is independent of $t$.

Combining these estimates, we have $\left|K\left(t, x, x^{\prime}\right)\right|>c_{1} t^{-\frac{1}{3}}-2 C t^{-\frac{1}{2}}>c_{1} / 2 t^{-\frac{1}{3}}$ for any $\left(x, x^{\prime}\right)$ satisfying $\left(x-x^{\prime}\right) / t=\tau_{0}$, which implies (5.1).

Second, suppose there are other $a_{1}, a_{2} \in I$ such that $a_{1}, a_{2}, a_{0}$ are distinct and $\partial_{a} F_{\tau_{0}}(a), \partial_{a}^{2} F_{\tau_{0}}(a)$ both vanish at $a=a_{1}, a_{2}$. Then $P(x)$ vanishes at $\wp\left(a_{j}\right)$, $j=0,1,2$.

Since $a_{0} \in\left(\omega_{1}, \omega_{2}\right)$, we have that $-i \wp^{\prime}\left(a_{0}\right)>0$ and $\zeta(\omega) / \omega+\wp\left(a_{0}\right)>0$ by Lemma 2.4. Thus $\tau_{0}<0$ by (3.4). Similar analysis shows that when $a \in$ $\left(\omega_{2}, \omega_{2}+i \omega^{\prime}\right) \cup\left(i \omega^{\prime}, 2 i \omega^{\prime}\right), \partial_{a} F_{\tau_{0}}(a) \neq 0$. Therefore, $a_{1}, a_{2} \in\left(0, i \omega^{\prime}\right)$. 
Thus $\wp\left(a_{j}\right), j=0,1,2$ are distinct and are the three roots of $P(x)$. This implies that there is no other $a \in I$ such that $\partial_{a} F_{\tau_{0}}(a)=\partial_{a}^{2} F_{\tau_{0}}(a)=0$.

We again set $\delta>0$ small enough such that for $a \notin \bigcup_{j=0}^{3}\left(a_{j}-\delta, a_{j}+\delta\right) \subset I$, $\left|\partial_{a} F_{\tau_{0}}(a)\right|+\left|\partial_{a}^{2} F_{\tau_{0}}(a)\right|$ is uniformly greater than some positive constant. Given any large $t$, take $\left(x, x^{\prime}\right)$ such that $\frac{x-x^{\prime}}{t}=\tau_{0}$. The earlier argument implies

$$
K\left(t, x, x^{\prime}\right)=\sum_{j=0}^{2} \int_{I} e^{-i t F_{\tau_{0}}(a)} \rho_{j}(a) m_{a}(x) m_{-a}\left(x^{\prime}\right) \frac{-\wp^{\prime}(a) d a}{W(a)}+O\left(t^{-\frac{1}{2}}\right),
$$

where $\rho_{j}(a)=1$ when $\left|a-a_{j}\right|<\delta$ and $\rho_{j}(a)=0$ when $\left|a-a_{j}\right|>2 \delta$.

By Lemma 5.1,

$$
K\left(t, x, x^{\prime}\right)=a_{3} t^{-\frac{1}{3}} \sum_{j=0}^{2}\left(F_{\tau_{0}}^{(3)}\left(a_{i}\right)\right)^{-\frac{1}{3}} m_{a_{j}}(x) m_{-a_{j}}\left(x^{\prime}\right) \frac{-\wp^{\prime}\left(a_{j}\right)}{W\left(a_{j}\right)}+O\left(t^{-\frac{1}{2}}\right) .
$$

Recall that $x$ and $x^{\prime}$ are related by $\left(x-x^{\prime}\right) / t=\tau_{0} \cdot m_{a_{j}}(x) m_{-a_{j}}\left(x^{\prime}\right), j=0,1,2$, are linearly independent as functions of $x \in \mathbb{R}$ and their nontrivial linear combination is a nonzero function. Therefore, there exist $x_{0}$ and $x_{0}^{\prime}$, satisfying $\left(x_{0}-x_{0}^{\prime}\right) / t=\tau_{0}$ and

$$
K\left(t, x_{0}, x_{0}^{\prime}\right)=c t^{-\frac{1}{3}}+O\left(t^{-\frac{1}{2}}\right),
$$

where $c$ is nonzero. Thus there exists $T$ such that for $t>T$

$$
\left|K\left(t, x_{0}, x_{0}^{\prime}\right)\right|>\frac{c}{2} t^{-\frac{1}{3}} .
$$

Finally, suppose that $a_{1}=a_{2}$ in the second case, which is equivalent to that $\wp\left(a_{1}\right)$ is a double root of $P(x)$ in $\left(-\infty, \wp\left(\omega_{3}\right)\right)$. Similarly, we have for $t>T$

$$
\begin{aligned}
K\left(t, x, x^{\prime}\right)= & \sum_{j=0}^{1} \int_{I} e^{-i t F_{\tau_{0}}(a)} \rho_{j}(a) m_{a}(x) m_{-a}\left(x^{\prime}\right) \frac{-\wp^{\prime}(a) d a}{W(a)}+O\left(t^{-\frac{1}{2}}\right) \\
= & a_{3} t^{-\frac{1}{3}}\left(F_{\tau_{0}}^{(3)}\left(a_{0}\right)\right)^{-\frac{1}{3}} m_{a_{0}}(x) m_{-a_{0}}\left(x^{\prime}\right) \frac{-\wp^{\prime}\left(a_{0}\right)}{W\left(a_{0}\right)}+ \\
& a_{4} t^{-\frac{1}{4}}\left(F_{\tau_{1}}^{(4)}\left(a_{1}\right)\right)^{-\frac{1}{4}} m_{a_{1}}(x) m_{-a_{1}}\left(x^{\prime}\right) \frac{-\wp^{\prime}\left(a_{1}\right)}{W\left(a_{1}\right)}+O\left(t^{-\frac{1}{2}}\right) .
\end{aligned}
$$

Therefore, there exists $\left(x_{0}, x_{0}^{\prime}\right)$ such that $\left|K\left(t, x, x^{\prime}\right)\right|>c t^{-\frac{1}{4}}>c t^{-\frac{1}{3}}$. This completes the proof of (5.1).

Our proof also gives the optimality of (1.12) in the case that $P(x)$ has a double root in $\left(-\infty, \wp\left(\omega_{3}\right)\right]$.

By the proof of Lemma 2.4, we see that when $g_{2} / 12 \geq(\zeta(\omega) / \omega)^{2}, P(x)$ has no double root in $\left(-\infty, \wp\left(\omega_{3}\right)\right.$ ]. Therefore, Corollary 1.2 holds.

Set $\omega=1$; it follows from Eq.(6.1) and (6.3) that $\frac{g_{2}}{12}-(\zeta(\omega) / \omega)^{2}$, as a function of $\omega^{\prime}>0$, is analytic and $\omega^{\prime}=0$ is its essential singular point. Numerical experiment indicates that $g_{2} / 12-(\zeta(\omega) / \omega)^{2} \approx 0.966104$ when $\omega=1$ and $\omega^{\prime}>5$. When $\omega=1$ and $\omega^{\prime} \rightarrow 0^{+}, g_{2} / 12-(\zeta(\omega) / \omega)^{2}$ assumes each real number infinitely many times. 


\section{Appendix}

Here we list some elementary properties of Weierstrass functions ([WW], [Ch], $[\mathbf{A k}],[\mathbf{G H}])$. A doubly-periodic function which is meromorphic is called an elliptic function. Suppose that $2 \omega_{1}$ and $2 \omega_{3}$ are two periods of an elliptic function $f(z)$ and $\Im\left(\omega_{3} / \omega_{1}\right) \neq 0$. Join in succession the points $0,2 \omega_{1}, 2 \omega_{1}+2 \omega_{3}, 2 \omega_{3}, 0$ and we obtain a parallelogram. If there is no point $\omega$ inside or on the boundary of this parallelogram (the vertices excepted) such that $f(z+\omega)=f(z)$ for all values of $z$, this parallelogram is called a fundamental period-parallelogram for an elliptic function with periods $2 \omega_{1}$ and $2 \omega_{3}$. As a set, we assume this parallelogram only includes one of four vertices and two edges adjacent to it. In this way, the $z$-plane can be covered with the translations of this parallelogram without any overlap. It can be shown that for any $c \in \mathbb{C}$, the number of roots (counting multiplicity) of the equation

$$
f(z)=c
$$

which lie in the fundamental period-parallelogram does not depend on $c$. This number is called the order of the elliptic function $f(z)$ and it equals the number of poles of $f$ inside a fundamental period-parallelogram.

Given $\omega_{1}, \omega_{3} \in \mathbb{C}$ with $\Im\left(\omega_{3} / \omega_{1}\right) \neq 0$, the Weierstrass elliptic function is defined as

$$
\wp(z)=\frac{1}{z^{2}}+\sum_{(m, n) \neq(0,0)}\left\{\left(z-2 m \omega_{1}-2 n \omega_{3}\right)^{-2}-\left(2 m \omega_{1}+2 n \omega_{3}\right)^{-2}\right\} .
$$

The summation extends over all integer values of $m$ and $n$, simultaneous zero values of $m$ and $n$ excepted. $\wp(z)$ is doubly-periodic, namely

$$
\wp(z)=\wp\left(z+2 \omega_{1}\right)=\wp\left(z+2 \omega_{3}\right) .
$$

$\wp(z)$ is an elliptic function of order 2 , with poles $\Omega_{m, n}=2 m \omega_{1}+2 n \omega_{3}$. Each pole $\Omega_{m, n}$ is of degree 2. $\wp(z)$ is an even function, $\wp(z)=\wp(-z)$. The Laurent's expansion of $\wp(z)$ at $z=0$ is written as

$$
\wp(z)=z^{-2}+\frac{1}{20} g_{2} z^{2}+\frac{1}{28} g_{3} z^{4}+O\left(z^{6}\right),
$$

where $g_{2}, g_{3}$ are the constants in Eq.(1.5) and (1.6). Explicitly, we have

$$
g_{2}=60 \sum_{(m, n) \neq(0,0)} \Omega_{m, n}^{-4}, \quad g_{3}=140 \sum_{(m, n) \neq(0,0)} \Omega_{m, n}^{-6} .
$$

Here $g_{2}$ and $g_{3}$ are called the invariants of $\wp$ and they uniquely characterize $\wp$.

Since $\wp^{\prime}$ is odd and elliptic of order 3 , it has three zeroes in its fundamental period-parallelogram. It is clear that these zeroes are the half periods $\omega_{1}, \omega_{2}=$ $\omega_{1}+\omega_{3}$ and $\omega_{3}$. Denote $e_{j}=\wp\left(\omega_{j}\right), j=1,2,3$. The fact that $\wp(z)$ is of order 2 implies that $e_{1}, e_{2}, e_{3}$ are distinct and that $\wp^{\prime \prime}$ does not vanish at $\omega_{j}, j=1,2,3$. Furthermore, Eq.(1.5) implies that $e_{1}, e_{2}, e_{3}$ are the roots of the cubic polynomial

$$
4 x^{3}-g_{2} x-g_{3}=0 .
$$

The function $\zeta(z)$ is defined by the equation 


$$
\frac{d}{d z} \zeta(z)=-\wp(z)
$$

coupled with the condition $\lim _{z \rightarrow 0}\left(\zeta(z)-z^{-1}\right)=0 . \zeta(z)$ may also be represented as

$$
\zeta(z)=\frac{1}{z}+\sum_{(m, n) \neq(0,0)}\left\{\frac{1}{z-2 m \omega_{1}-2 n \omega_{3}}+\frac{1}{2 m \omega_{1}+2 n \omega_{3}}+\frac{z}{\left(2 m \omega_{1}+2 n \omega_{3}\right)^{2}}\right\} .
$$

$\zeta(z)$ is an odd meromorphic function of $z$ over the whole complex plane except at simple poles $\Omega_{m, n}$. The residue at each pole is 1 .

Write $\zeta\left(\omega_{1}\right)=\eta_{1}$ and $\zeta\left(\omega_{3}\right)=\eta_{3}$; then

$$
\eta_{1} \omega_{3}-\eta_{3} \omega_{1}=\frac{1}{2} \pi i
$$

$\zeta(z)$ is not doubly-periodic, however, it satisfies the following equations

$$
\zeta\left(z+2 \omega_{1}\right)=\zeta(z)+2 \eta_{1}, \quad \zeta\left(z+2 \omega_{3}\right)=\zeta(z)+2 \eta_{3} .
$$

Next we define $\sigma(z)$ by the equation

$$
\frac{d}{d z} \log \sigma(z)=\zeta(z)
$$

coupled with the condition $\lim _{z \rightarrow 0} \sigma(z) / z=1 . \sigma(z)$ is an odd entire function with simple zeroes at $\Omega_{m, n}$. Just like $\zeta(z), \sigma(z)$ satisfies

$$
\sigma\left(z+2 \omega_{1}\right)=-\sigma(z) e^{2 \eta_{1}\left(z+\omega_{1}\right)}, \quad \sigma\left(z+2 \omega_{3}\right)=-\sigma(z) e^{2 \eta_{1}\left(z+\omega_{3}\right)} .
$$

If we assume that $\omega_{1}=\omega, \omega_{3}=i \omega^{\prime}$ and $\omega, \omega^{\prime} \in \mathbb{R}$, then by symmetry $\wp(z)$ is real valued when $\Re z \in\left\{0, \omega_{1}\right\}$ or $\Im z \in\left\{0, i \omega_{3}\right\}$. $\zeta(z)$ is real valued on the real line and is pure imaginary when $\Re z=0$. Let $D$ to be the rectangle with vertices $0, \omega, \omega+i \omega^{\prime}$ and $i \omega^{\prime}$. Then $\wp(z)$ sends $D$ to the upper half plane conformally. As $z$ moves clockwise on the boundary of $D$ both starting and ending at $0, \wp(z)$ varies from $-\infty$ to $\infty$. This implies that $\wp\left(i \omega^{\prime}\right)<\wp\left(\omega+i \omega^{\prime}\right)<\wp(\omega)$.

\section{References}

Ak. Akhiezer, N. I., Elements of the theory of elliptic functions. Translations of Mathematical Monographs, 79. American Mathematical Society, Providence, RI, (1990).

Ch. Chandrasekharan, K., Elliptic Functions. Grundlehren der Mathematischen Wissenschaften, 281. Springer-Verlag, Berlin, (1985).

DSS. Damanik, D., Sims, R., Stolz, G. Localization for one-dimensional, continuum, BernoulliAnderson models. Duke Math. J. 114 (2002), No. 1, 59-100.

DZ. Deift, P., Zhou, X., A steepest descent method for oscillatory Riemann-Hilbert problems. Asymptotics for the MKdV equation. Annals. of Math. 137 (1993), 295-368.

Ea. M.S.P. Eastham Spectral Theory of Periodic Differential Equations. Scottish Academic Press, Edinburgh, (1973)

Er. Erdelyi, A., et al. Higher Transcendental Functioans, Vol. 3 McGraw-Hill, New York, (1955).

GH. Gesztesy, F., Holden, H. Soliton equations and their algebro-geometric solutions Vol. 1 (1+1)-Dimensional continuous models. Cambridge University Press, Cambridge, New York, (2003).

Go. Goldberg, M., Dispersive bounds for the three-dimensional Schrodinger equation with almost critical potentials. preprint (2004). 
KAIHUA CAI

GS. Goldberg, M., Schlag, W. Dispersive estimate for the Schrödinger operators in dimensions one and three. Comm. Math. Phys. 251, No.1, (2004), 157-178.

GSS. Gesztesy, F., Schweiger, W., Simon, B. Commutation methods applied to the $m K d V$ equation. Trans. Amer. Math. Soc. 324, No.2, (1991), 465-525.

GT. Garnett, J., Trubowitz, E. Gaps and bands of one dimensional periodic Schrödinger operators. Comment. Math. Helvetici 59 (1984), 258-312.

Ho. Hochstadt, H. On the Characterization of a Hill's Equation from its Spectrum. Archive for Rational Mechanics and Analysis, 19 (1965), 353-362.

JK. Jensen, A., Kato, T. Spectral properties of Schrödinger operators and time-decay of the wave functions. Duke Math. J. 46 (1979), 583-611.

JSS. Journé, J.-L., Soffer, A., Sogge, C. D. Decay estimates for Schrödinger operators. Comm. Pure. Appl. Math. 44 No.5, (1991), 573-604.

KM. Kuznetsuv, E. A., Mikhailov, A.V. Stability of Stationary waves in nonlinear weakly dispersive media. Sov. Phys.-Jetp, 40 No.5, (1974), 855-859.

Ko. Korotyaev, E. The propagation of the waves in periodic media at large time. Asymptotic Analysis, 15 (1997), 1-24.

MW. Magnus, W., Winkler, S. Hill's Equation. Interscinece Publisher, New York-London (1966).

R. Rauch, J. Local decay of scattering solutions to Schrödinger's equation. Comm. Math. Phys. 61 (1978), 149-168.

RS. Rodnianski, I., Schlag, W. Time decay of solutions of Schrödinger equations with rough and time dependent potentials. Invent. Math. 155 (2004), No. 3, 451-513.

St. Stein, E. Harmonic Analysis: Real-Variable Methods, Orthogonality, and Oscillatory Integrals. Princeton Univ. Press, (1993).

We. Weder, R. The $W_{k, p}$-continuity of the Schrödinger Wave Operators on the Line. Comm. Math. Phys. 208 (1999), 507-520.

We2. Weder, R. $L^{p} \rightarrow L^{p^{\prime}}$ estimates for the Schrödinger equation on the line and inverse scattering for the nonlinear Schrödinger equation with a potential. Journal of Functional Analysis 170 (2000), 37-68.

WW. Whittaker, E. T., Watson, G. N. A Course of Mordern Analysis. University Press, Cambridge, (1952).

Ya. Yajima, K. The $W^{k, p}$-continuity of wave operators for Schrödinger operators. J. Math. Soc. Japan 47 No. 3 (1995), 551-581.

Mathematical Science Research Institute, 17 Gauss Way, Berkeley, CA 94720-5070 E-mail address: kcai@msri.org 